\title{
RETRIEVAL OF A GREEN'S FUNCTION WITH REFLECTIONS FROM PARTLY COHERENT WAVES GENERATED BY A WAVE PACKET USING CROSS CORRELATIONS
}

\author{
MAARTEN V. DE HOOP*, JOSSELIN GARNIER ${ }^{\dagger}$, SEAN F. HOLMAN*, AND KNUT SØLNA
}

\begin{abstract}
We analyze the "field-field" cross correlation associated with partly coherent scattered waves generated by a wave packet. The configuration consists of a slab in which random medium fluctuations occur; the bottom of the slab is bounded by a deterministic discontinuity (a smooth reflector). Following the dyadic parabolic scaling of wave packets, and scaling the random fluctuations appropriately, we arrive at a description in terms of a system of linear Itô-Schrödinger diffusion models. Studying the Wigner distributions of the fields generated by these models, leads to a "blurring" transformation providing a complete characterization of the cross correlation. We obtain a description of the cross correlation in terms of this transformation applied to the effective transmission operator arising as a solution of the Itô-Schrödinger model. Most of the analysis is focussed on the interaction of the deterministic reflector with the random fluctuations through the waves in the regime of fine scales.
\end{abstract}

1. Introduction. We analyze the "field-field" cross correlation associated with partly coherent waves generated by a wave packet and observed at a pair of distinct receivers embedded within a random medium. The result is a Green's function estimate which captures both the high-frequency body wave reflections and the scattering due to the random fluctuations of the medium.

As a model configuration, we consider a slab in which random medium fluctuations occur. The bottom of the slab is bounded by a deterministic discontinuity (a smooth reflector). We consider waves incident from above the slab, and place our receivers within the slab to study the cross correlations between them. We assume that the deterministic component of the medium is constant within the slab and that the slab is flat.

The waves are excited by a wave packet. Wave packets contain a particular scale, which is selected in relation to the scale of the random fluctuations of the medium within the slab. Through localization in phase space, the propagation and scattering of a wave packet can be described by a coupled system of paraxial wave equations. The accuracy of this description can be proven to improve with increasingly finer scales, which is the regime considered here. Indeed, we view the cross correlations in the context of parametrix constructions. The paraxial form of the system allows for the use of Itô's stochastic calculus (for Hilbert-space valued processes) to analyze the scattering due to the random fluctuations; indeed, it enables the closure of the hierarchy of moment equations.

The solution procedure of each coupled system of paraxial wave equations is based on an invariant embedding type approach, generating a transmission and a reflection operator capturing the scattering due to the random fluctuations in the medium. Thus we arrive at a coupled system of Riccati equations for the mentioned operator kernels. In the limit of fine scales (high "frequency"), we then obtain a decoupled system of linear Itô-Schrödinger equations for formally limiting transmission and reflection operator kernels. We remark that the kernels themselves do not in general converge; however, the associated wave fields converge weakly as described in Proposition 4.1 below. The Itô-Schrödinger equations can be viewed as a generalization of the paraxial equations associated with a wave packet. By combining wave packets and the relevant solutions to the Itô-Schrödinger equations, we can generate general sources and the corresponding waves.

The solutions to the Itô-Schrödinger equations allow us to calculate the statistics of the transmitted and backscattered fields and analyze the cross correlations. Indeed, we find that the cross correlation of the wave field between two points has four primary contributions corresponding to waves directly transmitted through the two points, and those reflected. Each of these contributions can be represented as a filter with a self averaging property applied to the transmission operator

\footnotetext{
${ }^{1}$ Center for Computational and Applied Mathematics, Purdue University, 150 N. University Street, West Lafayette IN 47907, USA

${ }^{2}$ Laboratoire de Probabilités et Modèles Aléatoires \& Laboratoire Jacques-Louis Lions, Université Paris 7, site Chevaleret, case 7012, 75205 Paris Cedex 13, France

${ }^{3}$ Department of Mathematics, University of California at Irvine, Irvine, CA 92697-3875, USA, We thank the Mathmatical Sciences Research Institute Fall Program 2011 on Inverse Problems and Applications for partial support.
} 
arising from the Itô-Schrödinger equations. We estimate these filters in particular scaling regimes by analyzing Wigner distributions and thus obtain a description of the cross correlation in terms of a transformation applied to the transmission operator. The transformations represent diffusion or blurring. Essentially, by synthesizing a wave packet source and "field-field" cross correlation we succeed in estimating a Green's function from noisy reflections in the high-frequency regime. We explicitly characterize the reflections in the case of small offsets between the two detectors using the mentioned transformations in the strongly cluttered regime. Locally transverse medium diversity aids in sharpening these tranformations, while the wave packets accommodate uncertainty in phase space.

In the past decade, the understanding of how cross correlations between diffuse fields can recapture the Green's function has been an important topic of research [11, 14]. Cross correlating diffuse coda waves $[2]$ and ambient seismic noise $[10,15]$ resulted in the retrieval of surface waves observed at one station and excited at the other station. Furthermore, turning body waves have been observed in cross correlating ambient noise [9]; in an exploration seismology setting, reflected body waves have also been recovered by cross correlations [4]. Note that here we do not consider the ambient random noise case, rather a single localized source, however, we model the cluttered medium as a random field. We show that also in this configuration the cross correlation gives an estimate of the Green's function and that the estimate is enhanced by medium clutter. The exploitation of a scattering medium in capturing the Green's function by the "field-field" cross correlation in the case with ambient noise was studied in $[3,5]$. A study of point diffraction, in the absence of random medium fluctuations, but with a closed surface of point sources, in terms of the optical theorem, was given in [13]. The retrieval of direct and reflected body waves using a teleseismic ( $S$-wave) coda was discussed in [12]. We remark that the mathematical analysis of the "field-field" cross correlation in this setting from the point of view of stochastic calculus has just begun $[5,1,8]$.

2. Scaling and assumptions. We study the acoustic wave equation with a forcing term:

$$
\frac{\partial^{2} p}{\partial t^{2}}(t, z, \boldsymbol{x})-c^{2}(z, \boldsymbol{x}) \Delta p(t, z, \boldsymbol{x})=-\chi_{\epsilon}(t, \boldsymbol{x}) \delta^{\prime}(z),
$$

where $t \in \mathbb{R},(z, \boldsymbol{x}) \in \mathbb{R} \times \mathbb{R}^{d}$, and the Laplacian is taken in all of the spatial variables $(z, \boldsymbol{x})$. We distinguish $z$ as the special direction along which our waves will propagate. The wave speed $c(z, \boldsymbol{x})$ of the randomly heterogeneous medium is assumed to be

$$
c^{2}(z, \boldsymbol{x})=\left\{\begin{array}{cc}
1 & \text { if } z>0 \\
1+\epsilon^{3} \nu\left(\frac{z}{\varepsilon^{2}}, \frac{\boldsymbol{x}}{\varepsilon^{2}}\right) & \text { if }-L<z \leq 0 \\
c_{0}^{2} & \text { if } z \leq-L
\end{array}\right.
$$

for constants $L$ and $c_{0}>0$. Here $\nu$ is a zero-mean, stationary random field with correlation length and standard deviation of order one which represents the random fluctuations in the medium. We write

$$
\begin{aligned}
C(z, \boldsymbol{x}) & =\mathbb{E}\left[\nu\left(z^{\prime}+z, \boldsymbol{x}^{\prime}+\boldsymbol{x}\right) \nu\left(z^{\prime}, \boldsymbol{x}^{\prime}\right)\right], \\
D(\boldsymbol{x}) & =\int_{-\infty}^{\infty} C(z, \boldsymbol{x}) d z .
\end{aligned}
$$

We also assume that $\nu$ satisfies strong mixing conditions in $z$.

The forcing functions $\chi_{\epsilon}(t, \boldsymbol{x})$ are given by $\chi_{\epsilon}(t, \boldsymbol{x})=\chi\left(\varepsilon^{-4} t, \varepsilon^{-2} \boldsymbol{x}\right)$ where $\chi$ is the real part of a function whose Fourier transform in both $t$ and $\boldsymbol{x}$ has support contained in the box $[1 / 2,2]_{\omega} \times$ $[-1 / \sqrt{2}, 1 / \sqrt{2}]_{\kappa}^{d}$. Our conventions and notation for the Fourier transform are

$$
\begin{aligned}
\chi(t, \boldsymbol{x}) & =\frac{1}{(2 \pi)^{d+1}} \iint \hat{\chi}(\omega, \boldsymbol{\kappa}) e^{-i(\omega t-\boldsymbol{\kappa} \cdot \boldsymbol{x})} d \omega d \boldsymbol{\kappa}=\frac{1}{2 \pi} \int \check{\chi}(\omega, \boldsymbol{x}) e^{-i \omega t} d \omega, \\
\hat{\chi}(\omega, \boldsymbol{\kappa}) & =\iint \chi(t, \boldsymbol{x}) e^{i(\omega t-\boldsymbol{\kappa} \cdot \boldsymbol{x})} d t d \boldsymbol{x}, \quad \check{\chi}(\omega, \boldsymbol{x})=\int \chi(t, \boldsymbol{x}) e^{i \omega t} d t
\end{aligned}
$$

What we have in mind here is that $\chi_{\epsilon}$ represents the real part of a wave packet at scale $k$ with $2^{k}=\varepsilon^{-4}$ oriented in the $t$ "direction". 
This scaling with $\varepsilon$ is consistent with the paraxial regime, which will be discussed in the next section, in as much as solutions of (2.1) with $\nu=0$ may be approximated up to an error of order $\varepsilon^{4}$ (this error would in general be only of order $\varepsilon^{2}$ if there was a non-constant background) by the solution of a decoupled system of paraxial equations. We point out the important aspects of the scaling:

- The transverse width, $R_{0}$, of the source function is of order $\varepsilon^{2}$.

- The correlation length or radius of the fluctuations is of the same order as $R_{0}$; this regime guarantees non-trivial interaction between the fluctuations of the medium and the waves;

- The propagation distance $L$, is not scaled; thus the ratio between the propagation distance and the correlation length of the fluctuations is of order $\varepsilon^{-2}$

- The amplitude $\varepsilon^{3}$ of the fluctuations is chosen so as to obtain an effective limit of order one when $\varepsilon$ goes to zero. That is, if the magnitude of the fluctuations is smaller than $\varepsilon^{3}$, then the wave would propagate as if the medium were homogeneous, while if the order of magnitude is larger, then the wave would not penetrate the slab at all.

The objective here is to study how the random fluctuations affect observations (and in particular cross correlations) of the solution $p$ to (2.1) as $\varepsilon \rightarrow 0$ which represents the high frequency limit. Our main scaling assumption is thus:

Assumption 1. $\varepsilon \ll 1$.

3. Coupled system of paraxial equations, and transmission and reflection operators.

3.1. Totally deterministic case. In this subsection we consider only the totally deterministic case corresponding to $\nu=0$ in (2.1), so that the wave speed only depends on $z, c(z, \boldsymbol{x}) \equiv c(z)$ with $c(z)=1$ for $z>-L$ and $c(z)=c_{0}$ for $z<-L$. We provide precise estimates on how well the solutions of (2.1) can be approximated by solutions of a decoupled system of paraxial equations (ie. show that we are in the paraxial regime). Also, we study the transmitted and reflected wave fields in the high-frequency limit which is the deterministic version of Proposition 4.1.

To begin we convert (2.1) to an equivalent system which distinguishes $z$ in a more transparent way. Indeed, if $p^{\mathrm{D}}$ is a solution of (2.1), then we have a corresponding solution of the system

$$
\frac{\partial}{\partial z}\left(\begin{array}{l}
p^{\mathrm{D}} \\
v^{\mathrm{D}}
\end{array}\right)=\left(\begin{array}{cc}
0 & 1 \\
\frac{1}{c(z)^{2}} \frac{\partial^{2}}{\partial t^{2}}-\Delta_{\boldsymbol{x}} & 0
\end{array}\right)\left(\begin{array}{l}
p^{\mathrm{D}} \\
v^{\mathrm{D}}
\end{array}\right)+\left(\begin{array}{c}
0 \\
\chi_{\epsilon}(t, \boldsymbol{x}) \delta^{\prime}(z)
\end{array}\right),
$$

where $\Delta_{\boldsymbol{x}}$ is the transverse Laplacian. On the other hand, if $p^{\mathrm{D}}$ and $v^{\mathrm{D}}$ are solutions of (3.1) on $\{z \neq 0,-L\}$ satisfying the jump conditions

$$
p^{\mathrm{D}}\left(t, 0^{+}, \boldsymbol{x}\right)-p^{\mathrm{D}}\left(t, 0^{-}, \boldsymbol{x}\right)=\chi_{\epsilon}(t, \boldsymbol{x}) \quad \text { and } \quad v^{\mathrm{D}}\left(t, 0^{+}, \boldsymbol{x}\right)-v^{\mathrm{D}}\left(t, 0^{-}, \boldsymbol{x}\right)=0,
$$

and continuous across $z=-L$ then $p^{\mathrm{D}}$ is also a solution of (2.1).

We find solutions of (3.1) by diagonalizing the matrix operator on the right-hand side microlocally. Indeed, let $\lambda(\omega, \boldsymbol{\kappa})=\sqrt{\omega^{2}-|\boldsymbol{\kappa}|^{2}}$,

$$
\mathcal{Q}(\omega, \boldsymbol{\kappa})=\left(\begin{array}{cc}
\lambda^{-\frac{1}{2}} & \lambda^{-\frac{1}{2}} \\
i \lambda^{\frac{1}{2}} & -i \lambda^{\frac{1}{2}}
\end{array}\right), \quad \text { and } \quad \mathcal{Q}^{-1}(\omega, \boldsymbol{\kappa})=\frac{1}{2}\left(\begin{array}{cc}
\lambda^{\frac{1}{2}} & -i \lambda^{-\frac{1}{2}} \\
\lambda^{\frac{1}{2}} & i \lambda^{-\frac{1}{2}}
\end{array}\right) .
$$

It is straightforward to check that the unique solution to (3.1) on $\{-L<z<0\}$ with $p^{\mathrm{D}}\left(t, 0^{-}, \boldsymbol{x}\right)=$ $\varphi_{p}(t, \boldsymbol{x})$ and $v^{\mathrm{D}}\left(t, 0^{-}, \boldsymbol{x}\right)=\varphi_{v}(t, \boldsymbol{x})$ is given by

$$
\left(\begin{array}{c}
p^{\mathrm{D}}(\cdot, z, \cdot) \\
v^{\mathrm{D}}(\cdot, z, \cdot)
\end{array}\right)=\mathcal{P}^{\mathrm{D}}(z)\left(\begin{array}{c}
\varphi_{p} \\
\varphi_{v}
\end{array}\right)
$$

provided that the support of the Fourier transforms of $\varphi_{p}$ and $\varphi_{v}$ are compactly contained in the region $\{|\omega|>|\kappa|\}$, where the (deterministic) propagator is defined by

$$
\mathcal{P}^{\mathrm{D}}(z)\left(\begin{array}{l}
\varphi_{p} \\
\varphi_{v}
\end{array}\right):=\mathcal{Q}\left(D_{t}, D_{\boldsymbol{x}}\right)\left(\begin{array}{cc}
e^{i z \lambda\left(D_{t}, D_{\boldsymbol{x}}\right)} & 0 \\
0 & e^{-i z \lambda\left(D_{t}, D_{\boldsymbol{x}}\right)}
\end{array}\right) \mathcal{Q}^{-1}\left(D_{t}, D_{\boldsymbol{x}}\right)\left(\begin{array}{l}
\varphi_{p} \\
\varphi_{v}
\end{array}\right) .
$$


We specify $\varphi_{p}$ and $\varphi_{v}$ by invoking a directional decomposition of the wave field and requiring that there are no incoming waves. Indeed, let us suppose that $p^{\mathrm{D}}$ and $v^{\mathrm{D}}$ are our solutions of (3.1) and introduce the following directional decomposition:

$$
\left(\begin{array}{l}
a^{\mathrm{D}}(\cdot, z, \cdot) \\
b^{\mathrm{D}}(\cdot, z, \cdot)
\end{array}\right):=\left(\begin{array}{cc}
e^{-i z \lambda\left(D_{t}, D_{\boldsymbol{x}}\right)} & 0 \\
0 & e^{i z \lambda\left(D_{t}, D_{\boldsymbol{x}}\right)}
\end{array}\right) \mathcal{Q}^{-1}\left(D_{t}, D_{\boldsymbol{x}}\right)\left(\begin{array}{l}
p^{\mathrm{D}}(\cdot, z, \cdot) \\
v^{\mathrm{D}}(\cdot, z, \cdot)
\end{array}\right) .
$$

The first component $\left(a^{\mathrm{D}}\right)$ of the decomposition is the portion of the wave field traveling in the positive $z$ direction (up) while the second component $\left(b^{\mathrm{D}}\right)$ is the portion traveling in the negative $z$ direction (down). We ask that there are no incoming waves which thus means

$$
b^{\mathrm{D}}\left(t, 0^{+}, \boldsymbol{x}\right)=0 \quad \text { and } \quad a^{\mathrm{D}}\left(t,-L^{-}, \boldsymbol{x}\right)=0 .
$$

Using these conditions as well as (3.2) we obtain

$a^{\mathrm{D}}\left(t, 0^{+}, \boldsymbol{x}\right)=a^{\mathrm{D}}\left(t, 0^{-}, \boldsymbol{x}\right)+\frac{\sqrt{\lambda\left(D_{t}, D_{\boldsymbol{x}}\right)}}{2} \chi_{\varepsilon}(t, \boldsymbol{x}), \quad b^{\mathrm{D}}\left(t, 0^{-}, \boldsymbol{x}\right)=-\frac{\sqrt{\lambda\left(D_{t}, D_{\boldsymbol{x}}\right)}}{2} \chi_{\varepsilon}(t, \boldsymbol{x})$,

$a^{\mathrm{D}}\left(t,-L^{+}, \boldsymbol{x}\right)=e^{2 i L \lambda\left(D_{t}, D_{\boldsymbol{x}}\right)} \mathcal{R}^{\mathrm{D}}\left(D_{t}, D_{\boldsymbol{x}}\right) b^{\mathrm{D}}\left(t,-L^{+}, \boldsymbol{x}\right), \quad b^{\mathrm{D}}\left(t,-L^{-}, \boldsymbol{x}\right)=\mathcal{T}^{\mathrm{D}}\left(D_{t}, D_{\boldsymbol{x}}\right) b^{\mathrm{D}}\left(t,-L^{+}, \boldsymbol{x}\right)$,

where $\mathcal{R}^{\mathrm{D}}$ and $\mathcal{T}^{\mathrm{D}}$ are the frequency-dependent reflection and transmission operators of the interface at $z=-L$ :

$$
\mathcal{R}^{\mathrm{D}}(\omega, \boldsymbol{\kappa})=\frac{c_{0}-l_{0}(\omega, \boldsymbol{\kappa})}{c_{0}+l_{0}(\omega, \boldsymbol{\kappa})}, \quad \mathcal{T}^{\mathrm{D}}(\omega, \boldsymbol{\kappa})=2 \frac{\sqrt{c_{0} l_{0}(\omega, \boldsymbol{\kappa})}}{c_{0}+l_{0}(\omega, \boldsymbol{\kappa})}, \quad \text { and } \quad l_{0}(\omega, \boldsymbol{\kappa})=\sqrt{\frac{\omega^{2}-c_{0}^{2}|\boldsymbol{\kappa}|^{2}}{\omega^{2}-|\boldsymbol{\kappa}|^{2}}} .
$$

By (3.2) and all of these conditions we have

$$
\begin{aligned}
p^{\mathrm{D}}\left(t,-L^{-}, \boldsymbol{x}\right) & =-\frac{1}{2} e^{i L \lambda\left(D_{t}, D_{\boldsymbol{x}}\right)} \mathcal{T}^{\mathrm{D}}\left(D_{t}, D_{\boldsymbol{x}}\right) \chi_{\varepsilon}(t, \boldsymbol{x}), \\
p^{\mathrm{D}}\left(t, 0^{+}, \boldsymbol{x}\right) & =-\frac{1}{2} e^{2 i L \lambda\left(D_{t}, D_{\boldsymbol{x}}\right)} \mathcal{R}^{\mathrm{D}}\left(D_{t}, D_{\boldsymbol{x}}\right) \chi_{\varepsilon}(t, \boldsymbol{x})+\frac{1}{2} \chi_{\varepsilon}(t, \boldsymbol{x}) .
\end{aligned}
$$

Now we may establish the following elementary result about high-frequency transmission and reflection of the wave field.

Theorem 3.1. The pair $\left(p^{\mathrm{D}}\left(L+\varepsilon^{4} t,-L^{-}, \varepsilon^{2} \boldsymbol{x}\right), p^{\mathrm{D}}\left(2 L+\varepsilon^{4} t, 0^{+}, \varepsilon^{2} \boldsymbol{x}\right)-1 / 2 \chi\left(2 L / \varepsilon^{4}+t, \boldsymbol{x}\right)\right)$ converges in $C^{0}\left(\mathbb{R}_{t}, L^{2}\left(\mathbb{R}_{\boldsymbol{x}}^{d}, \mathbb{R}^{2}\right)\right) \cap L^{2}\left(\mathbb{R}_{t}, L^{2}\left(\mathbb{R}_{\boldsymbol{x}}^{d}, \mathbb{R}^{2}\right)\right)$ to $\left(p_{\mathrm{T}}^{\mathrm{D}}(t, \boldsymbol{x}), p_{\mathrm{R}}^{\mathrm{D}}(t, \boldsymbol{x})\right)$ as $\varepsilon \rightarrow 0$ where

$$
p_{\mathrm{T}}^{\mathrm{D}}(t, \boldsymbol{x})=-\frac{\mathcal{T}_{0}}{2} e^{-i L \frac{\left|D_{\boldsymbol{x}}\right|^{2}}{2 D_{t}}} \chi(t, \boldsymbol{x}), \quad p_{\mathrm{R}}^{\mathrm{D}}(t, \boldsymbol{x})=-\frac{\mathcal{R}_{0}}{2} e^{-i L \frac{\left|D_{\boldsymbol{x}}\right|^{2}}{D_{t}}} \chi(t, \boldsymbol{x}),
$$

and

$$
\mathcal{T}_{0}=2 \frac{c_{0}^{1 / 2}}{c_{0}+1}, \quad \mathcal{R}_{0}=\frac{c_{0}-1}{c_{0}+1}
$$

Proof. Note that the support of $\hat{\chi}_{\varepsilon}$ is contained in (for $\varepsilon$ sufficiently small) the set $\{\omega \geq$ $\left.|\boldsymbol{\kappa}|^{2}\right\} \cap\left\{\omega>1 /\left(2 \varepsilon^{4}\right)\right\}$. Thus $l_{0}(\omega, \boldsymbol{\kappa}) \rightarrow 1$ and $\lambda(\omega, \boldsymbol{\kappa})-\left(\omega-\frac{|\boldsymbol{\kappa}|^{2}}{2 \omega}\right) \rightarrow 0$ as $\varepsilon \rightarrow 0$ on this support. Together with Parseval's theorem this can be used to complete the proof.

Another way to write the formulas in Theorem 3.1 for $p_{\mathrm{T}}^{\mathrm{D}}$ and $p_{\mathrm{R}}^{\mathrm{D}}$ is

$$
\begin{aligned}
& \check{p}_{\mathrm{T}}^{\mathrm{D}}(\omega, \boldsymbol{x})=-\frac{\mathcal{T}_{0}}{4 \pi} \int \check{\mathcal{T}}\left(\omega,-L, 0, \boldsymbol{x}, \boldsymbol{x}^{\prime}\right) \check{\chi}\left(\omega, \boldsymbol{x}^{\prime}\right) d \boldsymbol{x}^{\prime}, \\
& \check{p}_{\mathrm{R}}^{\mathrm{D}}(\omega, \boldsymbol{x})=-\frac{\mathcal{R}_{0}}{4 \pi} \int \check{\mathcal{R}}\left(\omega,-L, 0, \boldsymbol{x}, \boldsymbol{x}^{\prime}\right) \check{\chi}\left(\omega, \boldsymbol{x}^{\prime}\right) d \boldsymbol{x}^{\prime},
\end{aligned}
$$


where the kernels of the operators $\check{\mathcal{R}}$ and $\check{\mathcal{T}}$ solve the equations

$$
\begin{aligned}
\frac{\partial}{\partial z} \check{\mathcal{R}}\left(\omega,-L, z, \boldsymbol{x}, \boldsymbol{x}^{\prime}\right) & =\frac{i}{2 \omega}\left(\Delta_{\boldsymbol{x}}+\Delta_{\boldsymbol{x}^{\prime}}\right) \check{\mathcal{R}}\left(\omega,-L, z, \boldsymbol{x}, \boldsymbol{x}^{\prime}\right), \\
\frac{\partial}{\partial z} \check{\mathcal{T}}\left(\omega,-L, z, \boldsymbol{x}, \boldsymbol{x}^{\prime}\right) & =\frac{i}{2 \omega} \Delta_{\boldsymbol{x}^{\prime}} \check{\mathcal{T}}\left(\omega,-L, z, \boldsymbol{x}, \boldsymbol{x}^{\prime}\right),
\end{aligned}
$$

subject to the condition that they are the kernels of the identity at $z=-L$. These should be compared with the diffusion models appearing in proposition 4.1. What we see is that the addition of the random fluctuations precisely adds the Brownian terms.

We now take a slightly different perspective and look at how well the solutions of (3.1) on $-L<z<0$ can be approximated by solutions of a decoupled system of paraxial equations. To accomplish this we replace each of the factors in (3.3) by an approximation. Firstly, as we shall soon see, it is in fact sufficient to use the zero order expansions of $\mathcal{Q}$ and $\mathcal{Q}^{-1}$ about $|\boldsymbol{\kappa}|^{2} / \omega^{2}=0$, which are given by

$$
\widetilde{\mathcal{Q}}(\omega)=\left(\begin{array}{cc}
\sqrt{\frac{1}{\omega}} & \sqrt{\frac{1}{\omega}} \\
i \sqrt{\omega} & -i \sqrt{\omega}
\end{array}\right) \quad \text { and } \quad \widetilde{\mathcal{Q}}^{-1}(\omega)=\frac{1}{2}\left(\begin{array}{cc}
\sqrt{\omega} & -i \sqrt{\frac{1}{\omega}} \\
\sqrt{\omega} & i \sqrt{\frac{1}{\omega}}
\end{array}\right) .
$$

We may also replace $\lambda$ by a second order expansion giving the following approximate solution of (3.1) on $\{-L<z<0\}$ with $p^{\mathrm{A}}\left(t, 0^{-}, \boldsymbol{x}\right)=\varphi_{p}(t, \boldsymbol{x})$ and $v^{\mathrm{A}}\left(t, 0^{-}, \boldsymbol{x}\right)=\varphi_{v}(t, \boldsymbol{x})$ :

$$
\left(\begin{array}{l}
p^{\mathrm{A}}(\cdot, z, \cdot) \\
v^{\mathrm{A}}(\cdot, z, \cdot)
\end{array}\right)=\mathcal{P}^{\mathrm{A}}(z)\left(\begin{array}{l}
\varphi_{p} \\
\varphi_{v}
\end{array}\right)
$$

where the approximate propagator is defined by

$$
\mathcal{P}^{\mathrm{A}}(z)\left(\begin{array}{l}
\varphi_{p} \\
\varphi_{v}
\end{array}\right):=\widetilde{\mathcal{Q}}\left(D_{t}\right)\left(\begin{array}{cc}
e^{i z D_{t}\left(1-\frac{\left|D_{\boldsymbol{x}}\right|^{2}}{2 D_{t}^{2}}\right)} & 0 \\
0 & e^{-i z D_{t}\left(1-\frac{\left|D_{\boldsymbol{x}}\right|^{2}}{2 D_{t}^{2}}\right)}
\end{array}\right) \widetilde{\mathcal{Q}}^{-1}\left(D_{t}\right)\left(\begin{array}{l}
\varphi_{p} \\
\varphi_{v}
\end{array}\right)
$$

We now have the following result for how well $\left(p^{\mathrm{A}}, v^{\mathrm{A}}\right)$ given in terms of the operator $\mathcal{P}^{\mathrm{A}}(z)$ approximates the actual solution $\left(p^{\mathrm{D}}, v^{\mathrm{D}}\right)$ given in terms of $\mathcal{P}^{\mathrm{D}}(z)$.

THEOREM 3.2. Suppose that $\widehat{\varphi_{p}}$ and $\widehat{\varphi_{v}}$ have compact support contained in the set $\left\{2 \omega-|\kappa|^{2}>\right.$ $0\} \cap\left\{\omega>1 / \varepsilon^{4}\right\}$ for a given $\varepsilon<1 / \sqrt{2}$. Then for $-L<z<0$ there is a constant $C$ depending only on $L$ such that

$$
\left\|\pi_{p} \circ\left(\mathcal{P}^{\mathrm{D}}(z)-\mathcal{P}^{\mathrm{A}}(z)\right)\left(\begin{array}{c}
\varphi_{p} \\
\varphi_{v}
\end{array}\right)\right\|_{L^{2}} \leq C\left(\varepsilon^{4}\left\|\varphi_{p}\right\|_{L^{2}}+\varepsilon^{8}\left\|\varphi_{v}\right\|_{L^{2}}\right)
$$

and

$$
\left\|\pi_{v} \circ\left(\mathcal{P}^{\mathrm{D}}(z)-\mathcal{P}^{\mathrm{A}}(z)\right)\left(\begin{array}{c}
\varphi_{p} \\
\varphi_{v}
\end{array}\right)\right\|_{L^{2}} \leq C\left(\left\|\varphi_{p}\right\|_{L^{2}}+\varepsilon^{4}\left\|\varphi_{v}\right\|_{L^{2}}\right)
$$

where $\pi_{p}$ and $\pi_{v}$ are the projections respectively onto the first and second components (that is, the "p" and "v" components $)$, and $L^{2}$ means $L^{2}\left(\mathbb{R}_{t} \times \mathbb{R}_{\boldsymbol{x}}^{d}, \mathbb{R}\right)$.

REMARK 3.3. The difference between the two estimates in the theorem reflects the fact that $v$ should in general have one degree less regularity than $p$.

Proof. On the support of $\widehat{\varphi_{p}}$ and $\widehat{\varphi_{v}}$ we can bound the differences between $e^{ \pm i \lambda}-e^{ \pm i \omega\left(1-\frac{|\kappa|^{2}}{2 \omega^{2}}\right)}$ and $\lambda^{1 / 2}-\sqrt{\omega}$ in terms of $\varepsilon$. Doing so and applying Parseval's theorem gives the estimates. $\square$

Additionally we can also introduce a directional decomposition of the approximate solution

$$
\left(\begin{array}{l}
a^{\mathrm{A}}(\cdot, z, \cdot) \\
b^{\mathrm{A}}(\cdot, z, \cdot)
\end{array}\right)=\left(\begin{array}{cc}
e^{-i z D_{t}} & 0 \\
0 & e^{i z D_{t}}
\end{array}\right) \widetilde{\mathcal{Q}}^{-1}\left(D_{t}\right) \mathcal{P}^{\mathrm{A}}(z)\left(\begin{array}{l}
\varphi_{p} \\
\varphi_{v}
\end{array}\right)
$$


It is easy to check that this decomposition satisfies the decoupled equations

$$
\begin{aligned}
\frac{\partial \check{a}^{\mathrm{A}}}{\partial z} & =\frac{i}{2 \omega} \Delta_{\boldsymbol{x}} \check{a}^{\mathrm{A}}, \\
\frac{\partial \check{b}^{\mathrm{A}}}{\partial z} & =-\frac{i}{2 \omega} \Delta_{\boldsymbol{x}} \check{b}^{\mathrm{A}} .
\end{aligned}
$$

Thus by Theorem 3.2 we can approximate solutions $p$ of the original equation (2.1) up to accuracy $\approx \varepsilon^{4}$ by solving these decoupled paraxial equations.

3.2. Riccati equations. We use the decomposition (3.10) for the approximate solution, also in the presence of random fluctuations. Indeed if $p$ and $v$ denote our solution of (2.1) with the fluctuations, then

$$
\left(\begin{array}{l}
a \\
b
\end{array}\right)(t, z, \boldsymbol{x}):=\left(\begin{array}{cc}
e^{-i z D_{t}} & 0 \\
0 & e^{i z D_{t}}
\end{array}\right) \widetilde{\mathcal{Q}}^{-1}\left(D_{t}\right)\left(\begin{array}{l}
p \\
v
\end{array}\right)(t, z, \boldsymbol{x})
$$

is our directional decomposition. This is actually only an approximation of the true decomposition given by (3.5). It is also convenient to rescale $a$ and $b$ :

$$
a^{\varepsilon}(t, z, \boldsymbol{x})=a\left(\varepsilon^{4} t, z, \varepsilon^{2} \boldsymbol{x}\right), \quad b^{\varepsilon}(t, z, \boldsymbol{x})=b\left(\varepsilon^{4} t, z, \varepsilon^{2} \boldsymbol{x}\right),
$$

and work in the frequency domain by considering $\check{a}^{\varepsilon}$ and $\check{b}^{\varepsilon}$. Using (2.1) we obtain the following exact system for $-L<z<0$ :

$$
\begin{aligned}
& \frac{\partial \check{a}^{\varepsilon}}{\partial z}=\left(\frac{i \omega}{2 \varepsilon} \nu\left(\frac{z}{\varepsilon^{2}}, \boldsymbol{x}\right)+\frac{i}{2 \omega} \Delta_{\boldsymbol{x}}\right) \check{a}^{\varepsilon}+e^{-2 i \omega \frac{z}{\varepsilon^{4}}}\left(\frac{i \omega}{2 \varepsilon} \nu\left(\frac{z}{\varepsilon^{2}}, \boldsymbol{x}\right)+\frac{i}{2 \omega} \Delta_{\boldsymbol{x}}\right) \check{b}^{\varepsilon}, \\
& \frac{\partial \check{b}^{\varepsilon}}{\partial z}=-e^{2 i \omega \frac{z}{\varepsilon^{4}}}\left(\frac{i \omega}{2 \varepsilon} \nu\left(\frac{z}{\varepsilon^{2}}, \boldsymbol{x}\right)+\frac{i}{2 \omega} \Delta_{\boldsymbol{x}}\right) \check{a}^{\varepsilon}-\left(\frac{i \omega}{2 \varepsilon} \nu\left(\frac{z}{\varepsilon^{2}}, \boldsymbol{x}\right)+\frac{i}{2 \omega} \Delta_{\boldsymbol{x}}\right) \check{b}^{\varepsilon} .
\end{aligned}
$$

We approximate the condition that there are no incoming waves by asking that $\check{a}^{\varepsilon}\left(\omega,-L^{-}, \boldsymbol{x}\right)=0$ and $\breve{b}^{\varepsilon}\left(\omega, 0^{+}, \boldsymbol{x}\right)=0$. This leads to the boundary and jump conditions:

$$
\begin{gathered}
\check{b}^{\varepsilon}\left(\omega, 0^{-}, \boldsymbol{x}\right)=-\frac{\sqrt{\omega}}{2 \varepsilon^{2}} \check{\chi}(\omega, \boldsymbol{x}), \quad \check{a}^{\varepsilon}\left(\omega,-L^{+}, \boldsymbol{x}\right)=\mathcal{R}_{0} e^{2 i \omega \frac{L}{\varepsilon^{4}}} \check{b}^{\varepsilon}\left(\omega,-L^{+}, \boldsymbol{x}\right), \\
\check{b}^{\varepsilon}\left(\omega,-L^{-}, \boldsymbol{x}\right)=\mathcal{T}_{0} \check{b}^{\varepsilon}\left(\omega,-L^{+}, \boldsymbol{x}\right), \quad \check{a}^{\varepsilon}\left(\omega, 0^{+}, \boldsymbol{x}\right)=\check{a}^{\varepsilon}\left(\omega, 0^{-}, \boldsymbol{x}\right)+\frac{\sqrt{\omega}}{2 \varepsilon^{2}} \check{\chi}(\omega, \boldsymbol{x}),
\end{gathered}
$$

where $\mathcal{R}_{0}$ and $\mathcal{T}_{0}$ are the same as in (3.7). Indeed, in view of Theorem 3.2 with $\nu=0$ we expect decoupling of (3.11)-(3.12) with solutions accurate up to order $\varepsilon^{4}$.

To capture the transmission and reflection of the wave field in the presence of fluctuations we apply an invariant imbedding approach to obtain a representation valid for $-L<z<0$ :

$$
\begin{aligned}
\check{b}^{\varepsilon}\left(\omega,-L^{-}, \boldsymbol{x}\right) & =\mathcal{T}_{0} \int \check{\mathcal{T}}^{\varepsilon}\left(\omega,-L, z, \boldsymbol{x}, \boldsymbol{x}^{\prime}\right) \check{b}^{\varepsilon}\left(\omega, z, \boldsymbol{x}^{\prime}\right) d \boldsymbol{x}^{\prime}, \\
\check{a}^{\varepsilon}(\omega, z, \boldsymbol{x}) & =\mathcal{R}_{0} e^{2 i \omega \frac{L}{\varepsilon^{4}}} \int \check{\mathcal{R}}^{\varepsilon}\left(\omega,-L, z, \boldsymbol{x}, \boldsymbol{x}^{\prime}\right) \check{b}^{\varepsilon}\left(\omega, z, \boldsymbol{x}^{\prime}\right) d \boldsymbol{x}^{\prime},
\end{aligned}
$$

where the operators $\check{\mathcal{T}}^{\varepsilon}$ and $\check{\mathcal{R}}^{\varepsilon}$, defined through their kernels, satisfy a natural coupled system of operator Riccati equations which follow from the equations satisfied by the local amplitudes. Indeed, 
using the mode coupling equations (3.11-3.12) we find that

$$
\begin{aligned}
& \frac{\partial}{\partial z} \check{\mathcal{R}}^{\varepsilon}\left(\omega,-L, z, \boldsymbol{x}, \boldsymbol{x}^{\prime}\right)=\mathcal{R}_{0}^{-1} e^{-\frac{2 i \omega}{\varepsilon^{4}}(z+L)} \delta\left(\boldsymbol{x}-\boldsymbol{x}^{\prime}\right)\left(\frac{i \omega}{2 \varepsilon} \nu\left(\frac{z}{\varepsilon^{2}}, \boldsymbol{x}^{\prime}\right)+\frac{i}{2 \omega} \Delta_{\boldsymbol{x}^{\prime}}\right) \\
& \quad+\mathcal{R}_{0} e^{\frac{2 i \omega}{\varepsilon^{4}}(z+L)} \int \check{\mathcal{R}}^{\varepsilon}\left(\omega,-L, z, \boldsymbol{x}, \boldsymbol{x}_{1}\right)\left(\frac{i \omega}{2 \varepsilon} \nu\left(\frac{z}{\varepsilon}, \boldsymbol{x}_{1}\right)+\frac{i}{2 \omega} \Delta_{\boldsymbol{x}_{1}}\right) \check{\mathcal{R}}^{\varepsilon}\left(\omega,-L, z, \boldsymbol{x}_{1}, \boldsymbol{x}^{\prime}\right) d \boldsymbol{x}_{1} \\
& \quad+\left(\frac{i \omega}{2 \varepsilon} \nu\left(\frac{z}{\varepsilon^{2}}, \boldsymbol{x}\right)+\frac{i}{2 \omega} \Delta_{\boldsymbol{x}}\right) \check{\mathcal{R}}^{\varepsilon}\left(\omega,-L, z, \boldsymbol{x}, \boldsymbol{x}^{\prime}\right) \\
& \quad+\check{\mathcal{R}}^{\varepsilon}\left(\omega,-L, z, \boldsymbol{x}, \boldsymbol{x}^{\prime}\right)\left(\frac{i \omega}{2 \varepsilon} \nu\left(\frac{z}{\varepsilon^{2}}, \boldsymbol{x}^{\prime}\right)+\frac{i}{2 \omega} \Delta_{\boldsymbol{x}^{\prime}}\right) \\
& \frac{\partial}{\partial z} \check{\mathcal{T}}^{\varepsilon}\left(\omega,-L, z, \boldsymbol{x}, \boldsymbol{x}^{\prime}\right)=\check{\mathcal{T}}^{\varepsilon}\left(\omega,-L, z, \boldsymbol{x}, \boldsymbol{x}^{\prime}\right)\left(\frac{i \omega}{2 \varepsilon} \nu\left(\frac{z}{\varepsilon^{2}}, \boldsymbol{x}^{\prime}\right)+\frac{i}{2 \omega} \Delta_{\boldsymbol{x}^{\prime}}\right) \\
& \quad+\mathcal{R}_{0} e^{\frac{2 i \omega}{\varepsilon^{4}}(z+L)} \int \check{\mathcal{T}}^{\varepsilon}\left(\omega,-L, z, \boldsymbol{x}, \boldsymbol{x}_{1}\right)\left(\frac{i \omega}{2 \varepsilon} \nu\left(\frac{z}{\varepsilon}, \boldsymbol{x}_{1}\right)+\frac{i}{2 \omega} \Delta_{\boldsymbol{x}_{1}}\right) \check{\mathcal{R}}^{\varepsilon}\left(\omega,-L, z, \boldsymbol{x}_{1}, \boldsymbol{x}^{\prime}\right) d \boldsymbol{x}_{1} .
\end{aligned}
$$

This system is supplemented with the initial conditions at $z=-L$ :

$$
\check{\mathcal{R}}^{\varepsilon}\left(\omega,-L, z=-L, \boldsymbol{x}, \boldsymbol{x}^{\prime}\right)=\delta\left(\boldsymbol{x}-\boldsymbol{x}^{\prime}\right), \quad \check{\mathcal{T}}^{\varepsilon}\left(\omega,-L, z=-L, \boldsymbol{x}, \boldsymbol{x}^{\prime}\right)=\delta\left(\boldsymbol{x}-\boldsymbol{x}^{\prime}\right) .
$$

In the system (3.15-3.16) the first two parameters $-L$ and $\omega$ of $\check{\mathcal{R}}^{\varepsilon}$ and $\check{\mathcal{T}}^{\varepsilon}$ are frozen. Therefore the systems for different frequencies $\omega$ are not related from the analytic point of view, but they are not independent from the statistical point of view since the systems are written in terms of the same realization of the process $\nu$. It turns out that the correlation properties of the reflection and transmission operators play a crucial role in the asymptotic regime $\varepsilon \rightarrow 0$ as we will see below.

The transmission and reflection operators evaluated at $z=0$ carry all the relevant information about the random medium from the point of view of the transmitted wave $p^{\varepsilon}\left(t,-L^{-}, \boldsymbol{x}\right):=$ $p\left(\varepsilon^{4} t,-L^{-}, \varepsilon^{2} \boldsymbol{x}\right)$ and the reflected wave $p^{\varepsilon}\left(t, 0^{+}, \boldsymbol{x}\right):=p\left(\varepsilon^{4} t, 0^{+}, \varepsilon^{2} \boldsymbol{x}\right)$, which are our main quantities of interest. In the frequency domain they can be expressed as

$$
\begin{aligned}
\check{p}^{\varepsilon}\left(\omega, 0^{+}, \boldsymbol{x}\right) & =\frac{\varepsilon^{2}}{\sqrt{\omega}}\left\{e^{i \omega \frac{2 L}{\varepsilon^{4}}} \mathcal{R}_{0} \int \check{\mathcal{R}}^{\varepsilon}\left(\omega,-L, 0, \boldsymbol{x}, \boldsymbol{x}^{\prime}\right) \check{b}^{\varepsilon}\left(\omega, 0^{-}, \boldsymbol{x}^{\prime}\right) d \boldsymbol{x}^{\prime}-\check{b}^{\varepsilon}\left(\omega, 0^{-}, \boldsymbol{x}\right)\right\}, \\
\check{p}^{\varepsilon}\left(\omega,-L^{-}, \boldsymbol{x}\right) & =\frac{\varepsilon^{2}}{\sqrt{\omega}} e^{i \omega \frac{L}{\varepsilon^{4}}} \mathcal{T}_{0} \int \check{\mathcal{T}}^{\varepsilon}\left(\omega,-L, 0, \boldsymbol{x}^{\prime}, \boldsymbol{x}^{\prime}\right) \check{b}^{\varepsilon}\left(\omega, 0^{-}, \boldsymbol{x}^{\prime}\right) d \boldsymbol{x}^{\prime},
\end{aligned}
$$

with $\breve{b}^{\varepsilon}\left(\omega, 0^{-}, \boldsymbol{x}\right)=-\frac{\sqrt{\omega}}{2 \varepsilon^{2}} \check{\chi}(\omega, \boldsymbol{x})$. We will also need the following representation formula for the field $p^{\varepsilon}\left(t,-L_{1}, \boldsymbol{x}\right):=p\left(\varepsilon^{4} t,-L_{1}, \varepsilon^{2} \boldsymbol{x}\right)$ inside the heterogeneous region: for $-L<-L_{1}<0$ we have

$$
\begin{aligned}
\check{p}^{\varepsilon}\left(\omega,-L_{1}, \boldsymbol{x}\right)= & \frac{\varepsilon^{2}}{\sqrt{\omega}}\left\{e^{i \omega \frac{2 L-L_{1}}{\varepsilon^{4}}} \mathcal{R}_{0} \iint \check{\mathcal{R}}^{\varepsilon}\left(\omega,-L,-L_{1}, \boldsymbol{x}, \boldsymbol{x}^{\prime \prime}\right) \check{\mathcal{T}}^{\varepsilon}\left(\omega,-L_{1}, 0, \boldsymbol{x}^{\prime \prime}, \boldsymbol{x}^{\prime}\right) d \boldsymbol{x}^{\prime \prime} \check{b}^{\varepsilon}\left(\omega, 0^{-}, \boldsymbol{x}^{\prime}\right) d \boldsymbol{x}^{\prime}\right. \\
& \left.+e^{i \omega \frac{L_{1}}{\varepsilon^{4}}} \int \check{\mathcal{T}}^{\varepsilon}\left(\omega,-L_{1}, 0, \boldsymbol{x}^{\prime}, \boldsymbol{x}^{\prime}\right) \check{b}^{\varepsilon}\left(\omega, 0^{-}, \boldsymbol{x}^{\prime}\right) d \boldsymbol{x}^{\prime}\right\} .
\end{aligned}
$$

In the scaling regime $\varepsilon \rightarrow 0$ we are able to deduce from the system (3.15-3.16) a description in terms of effective white noise models for the transmission and reflection operators, at least on the level of moments. We describe this in the next section.

4. Itô-Schrödinger diffusion models for transmitted and reflected fields. We center according to the travel time associated with the deterministic medium component and define the transmitted and reflected pressure fields by

$$
\begin{aligned}
& p_{\mathrm{R}}^{\varepsilon}(s, \boldsymbol{x}):=p\left(2 L+\varepsilon^{4} s, 0^{+}, \varepsilon^{2} \boldsymbol{x}\right)-\frac{1}{2} \chi\left(2 L / \varepsilon^{4}+s, \boldsymbol{x}\right), \\
& p_{\mathrm{T}}^{\varepsilon}(s, \boldsymbol{x}):=p\left(L+\varepsilon^{4} s,-L^{-}, \varepsilon^{2} \boldsymbol{x}\right) .
\end{aligned}
$$

The field $p_{\mathrm{T}}^{\varepsilon}(s, \boldsymbol{x})$ is the field observed just below the bottom interface at $z=-L^{-}$and observed around the expected arrival time $L$; the field $p_{\mathrm{R}}^{\varepsilon}(s, \boldsymbol{x})$ is the field observed just above the top interface 
at $z=0^{+}$coming from the reflection off the boundary at $z=-L$, and observed around the expected arrival time $2 L$. Using (3.17-3.18) they can be written as

$$
\begin{aligned}
& p_{\mathrm{R}}^{\varepsilon}(s, \boldsymbol{x})=-\frac{\mathcal{R}_{0}}{4 \pi} \iint \check{\mathcal{R}}^{\varepsilon}\left(\omega,-L, 0, \boldsymbol{x}, \boldsymbol{x}^{\prime}\right) \check{\chi}\left(\omega, \boldsymbol{x}^{\prime}\right) d \boldsymbol{x}^{\prime} e^{-i \omega s} d \omega, \\
& p_{\mathrm{T}}^{\varepsilon}(s, \boldsymbol{x})=-\frac{\mathcal{T}_{0}}{4 \pi} \iint \check{\mathcal{T}}^{\varepsilon}\left(\omega,-L, 0, \boldsymbol{x}, \boldsymbol{x}^{\prime}\right) \check{\chi}\left(\omega, \boldsymbol{x}^{\prime}\right) d \boldsymbol{x}^{\prime} e^{-i \omega s} d \omega .
\end{aligned}
$$

These fields are now characterized via effective scaling limit models for the transmission and reflection operators:

Proposition 4.1. The processes $\left(p_{\mathrm{T}}^{\varepsilon}(s, \boldsymbol{x})\right)_{s \in \mathbb{R}, \boldsymbol{x} \in \mathbb{R}^{d}},\left(p_{\mathrm{R}}^{\varepsilon}(s, \boldsymbol{x})\right)_{s \in \mathbb{R}, \boldsymbol{x} \in \mathbb{R}^{d}}$ converge in distribution as $\varepsilon \rightarrow 0$ in the space $C^{0}\left(\mathbb{R}, L^{2}\left(\mathbb{R}^{d}, \mathbb{R}^{2}\right)\right) \cap L^{2}\left(\mathbb{R}, L^{2}\left(\mathbb{R}^{d}, \mathbb{R}^{2}\right)\right)$ to the limit processes $\left(p_{\mathrm{T}}(s, \boldsymbol{x})\right)_{s \in \mathbb{R}, \boldsymbol{x} \in \mathbb{R}^{d}}$, $\left(p_{\mathrm{R}}(s, \boldsymbol{x})\right)_{s \in \mathbb{R}, \boldsymbol{x} \in \mathbb{R}^{d}}$ given by

$$
\begin{aligned}
& p_{\mathrm{R}}(s, \boldsymbol{x})=-\frac{\mathcal{R}_{0}}{4 \pi} \iint \check{\mathcal{R}}\left(\omega,-L, 0, \boldsymbol{x}, \boldsymbol{x}^{\prime}\right) \check{\chi}\left(\omega, \boldsymbol{x}^{\prime}\right) d \boldsymbol{x}^{\prime} e^{-i \omega s} d \omega, \\
& p_{\mathrm{T}}(s, \boldsymbol{x})=-\frac{\mathcal{T}_{0}}{4 \pi} \iint \check{\mathcal{T}}\left(\omega,-L, 0, \boldsymbol{x}, \boldsymbol{x}^{\prime}\right) \check{\chi}\left(\omega, \boldsymbol{x}^{\prime}\right) d \boldsymbol{x}^{\prime} e^{-i \omega s} d \omega,
\end{aligned}
$$

where $\mathcal{T}_{0}$ and $\mathcal{R}_{0}$, are defined by (3.7). The kernels of the operators $\left(\check{\mathcal{R}}\left(\omega,-L, z, \boldsymbol{x}, \boldsymbol{x}^{\prime}\right)\right)_{z \in[-L, 0]}$ and $\left(\check{\mathcal{T}}\left(\omega,-L, z, \boldsymbol{x}, \boldsymbol{x}^{\prime}\right)\right)_{z \in[-L, 0]}$ are the solutions of the following Itô-Schrödinger diffusion models:

$$
\begin{aligned}
d \check{\mathcal{R}}\left(\omega,-L, z, \boldsymbol{x}, \boldsymbol{x}^{\prime}\right) & =\frac{i}{2 \omega}\left(\Delta_{\boldsymbol{x}}+\Delta_{\boldsymbol{x}^{\prime}}\right) \check{\mathcal{R}}\left(\omega,-L, z, \boldsymbol{x}, \boldsymbol{x}^{\prime}\right) d z \\
& +\frac{i \omega}{2} \check{\mathcal{R}}\left(\omega,-L, z, \boldsymbol{x}, \boldsymbol{x}^{\prime}\right) \circ\left(d B(z, \boldsymbol{x})+d B\left(z, \boldsymbol{x}^{\prime}\right)\right), \\
d \check{\mathcal{T}}\left(\omega,-L, z, \boldsymbol{x}, \boldsymbol{x}^{\prime}\right) & =\frac{i}{2 \omega} \Delta_{\boldsymbol{x}^{\prime}} \check{\mathcal{T}}\left(\omega,-L, z, \boldsymbol{x}, \boldsymbol{x}^{\prime}\right) d z \\
& +\frac{i \omega}{2} \check{\mathcal{T}}\left(\omega,-L, z, \boldsymbol{x}, \boldsymbol{x}^{\prime}\right) \circ d B\left(z, \boldsymbol{x}^{\prime}\right),
\end{aligned}
$$

with the initial conditions at $z=-L$

$$
\check{\mathcal{R}}\left(\omega,-L, z=-L, \boldsymbol{x}, \boldsymbol{x}^{\prime}\right)=\delta\left(\boldsymbol{x}-\boldsymbol{x}^{\prime}\right), \quad \check{\mathcal{T}}\left(\omega,-L, z=-L, \boldsymbol{x}, \boldsymbol{x}^{\prime}\right)=\delta\left(\boldsymbol{x}-\boldsymbol{x}^{\prime}\right) .
$$

The symbol $\circ$ stands for the Stratonovich stochastic integral, and $B(z, \boldsymbol{x})$ is a real-valued Brownian field with covariance

$$
\mathbb{E}\left[B\left(z_{1}, \boldsymbol{x}_{1}\right) B\left(z_{2}, \boldsymbol{x}_{2}\right)\right]=\min \left\{z_{1}, z_{2}\right\} D\left(\boldsymbol{x}_{1}-\boldsymbol{x}_{2}\right)
$$

Making use of (3.19) and of the semigroup property of the effective operators we also find that the joint law for the direct arrival to the two points of observation located at $\left(-L_{1}, \varepsilon^{2} \boldsymbol{x}_{1}\right)$ and $\left(-L_{2}, \varepsilon^{2} \boldsymbol{x}_{2}\right)$, with $-L \leq-L_{2} \leq-L_{1} \leq 0$, can be characterized by

$$
\begin{aligned}
p\left(L_{1}+\varepsilon^{4} s,-L_{1}, \varepsilon^{2} \boldsymbol{x}_{1}\right) & \simeq-\frac{1}{4 \pi} \iint \check{\mathcal{T}}\left(\omega,-L_{1}, 0, \boldsymbol{x}_{1}, \boldsymbol{y}_{1}\right) \check{\chi}\left(\omega, \boldsymbol{y}_{1}\right) d \boldsymbol{y}_{1} e^{-i \omega s} d \omega \\
p\left(L_{2}+\varepsilon^{4} s,-L_{2}, \varepsilon^{2} \boldsymbol{x}_{2}\right) \simeq & -\frac{1}{4 \pi} \iiint \check{\mathcal{T}}\left(\omega,-L_{2},-L_{1}, \boldsymbol{x}_{2}, \boldsymbol{y}_{2}\right) \check{\mathcal{T}}\left(\omega,-L_{1}, 0, \boldsymbol{y}_{2}, \boldsymbol{y}_{1}\right) \\
& \times \check{\chi}\left(\omega, \boldsymbol{y}_{1}\right) d \boldsymbol{y}_{1} d \boldsymbol{y}_{2} e^{-i \omega s} d \omega .
\end{aligned}
$$

We note that, for $-L \leq-L_{2} \leq-L_{1} \leq 0$, the operators $\check{\mathcal{T}}\left(\omega,-L,-L_{2}, \boldsymbol{x}, \boldsymbol{x}^{\prime}\right)$ and $\check{\mathcal{R}}\left(\omega,-L,-L_{2}, \boldsymbol{x}, \boldsymbol{x}^{\prime}\right)$ are statistically independent of $\check{\mathcal{T}}\left(\omega,-L_{2},-L_{1}, \boldsymbol{x}, \boldsymbol{x}^{\prime}\right)$ and $\check{\mathcal{R}}\left(\omega,-L_{2},-L_{1}, \boldsymbol{x}, \boldsymbol{x}^{\prime}\right)$, which we exploit in evaluating the cross correlation. We remark here also that the transmission operator over the sub-slab $\left(-L_{1}, 0\right)$ appears in both expressions in (4.10-4.11) and it is this pairing that will lead to an expression for the cross correlation in terms of a statistically stable filter or transformation below. The general statistical properties of the operators $\check{\mathcal{R}}, \check{\mathcal{T}}$ were studied in [6]. 


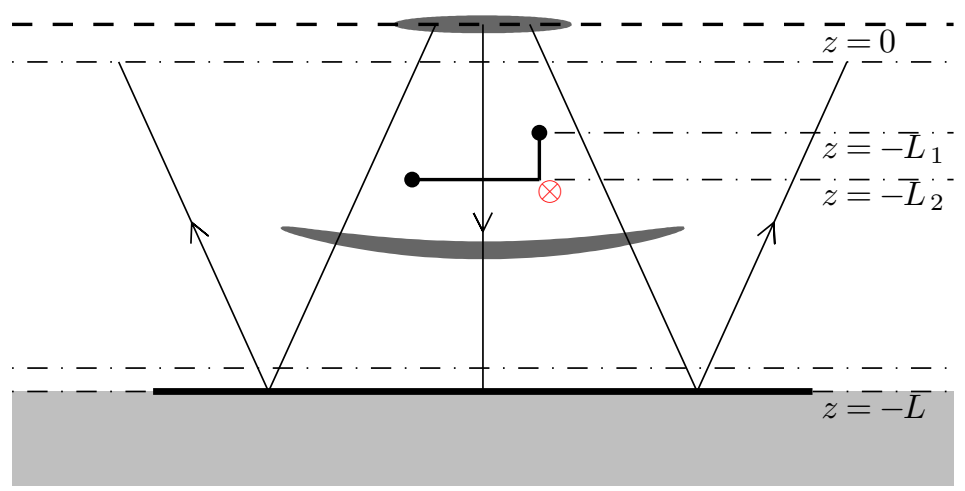

(a)

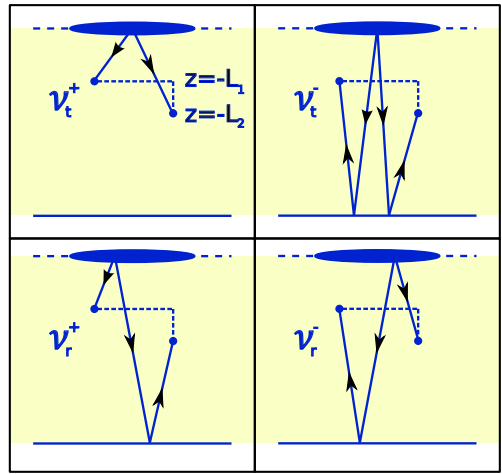

(b)

Fig. 5.1: (a) Configuration. The source is located at the surface $z=0$. An interface is present at depth $-L$. We compute the correlation in between the two points at depth $-L_{1}$ and $-L_{2}$. The random fluctuations occur in the interval $z \in(-L, 0)$. (b) The four contributions to the cross correlation between points $\left(-L_{1}, \varepsilon^{2} \boldsymbol{x}_{1}\right)$ and $\left(-L_{2}, \varepsilon^{2} \boldsymbol{x}_{2}\right)$.

We consider next the other main contributions to the field observations at $\left(-L_{1}, \varepsilon^{2} \boldsymbol{x}_{1}\right)$ and $\left(-L_{2}, \varepsilon^{2} \boldsymbol{x}_{2}\right)$, namely the ones associated with the wave propagating from the surface to the interface at $z=-L$ and back to the observation points. Again, making use of the semigroup property of the effective operators we find that the joint law for these "secondary" arrivals to the two points of observation located at $\left(-L_{1}, \varepsilon^{2} \boldsymbol{x}_{1}\right)$ and $\left(-L_{2}, \varepsilon^{2} \boldsymbol{x}_{2}\right)$, with $-L \leq-L_{2} \leq-L_{1} \leq 0$, can in our scaling regime be characterized by

$$
\begin{aligned}
p\left(2 L-L_{1}+\varepsilon^{4} s,-L_{1}, \varepsilon^{2} \boldsymbol{x}_{1}\right) \simeq & -\frac{\mathcal{R}_{0}}{4 \pi} \iiint \int \check{\mathcal{T}}\left(\omega,-L_{1},-L_{2}, \boldsymbol{x}_{1}, \boldsymbol{y}_{3}\right) \check{\mathcal{R}}\left(\omega,-L,-L_{2}, \boldsymbol{y}_{3}, \boldsymbol{y}_{2}\right) \\
& \times \check{\mathcal{T}}\left(\omega,-L_{2}, 0, \boldsymbol{y}_{2}, \boldsymbol{y}_{1}\right) \check{\chi}\left(\omega, \boldsymbol{y}_{1}\right) d \boldsymbol{y}_{1} d \boldsymbol{y}_{2} d \boldsymbol{y}_{3} e^{-i \omega s} d \omega, \\
p\left(2 L-L_{2}+\varepsilon^{4} s,-L_{2}, \varepsilon^{2} \boldsymbol{x}_{2}\right) \simeq & -\frac{\mathcal{R}_{0}}{4 \pi} \iiint \check{\mathcal{R}}\left(\omega,-L,-L_{2}, \boldsymbol{x}_{2}, \boldsymbol{y}_{2}\right) \check{\mathcal{T}}\left(\omega,-L_{2}, 0, \boldsymbol{y}_{2}, \boldsymbol{y}_{1}\right) \\
& \times \check{\chi}\left(\omega, \boldsymbol{y}_{1}\right) d \boldsymbol{y}_{1} d \boldsymbol{y}_{2} e^{-i \omega s} d \omega .
\end{aligned}
$$

We used the particular representation above to show how the transmission operator over the sub-slab $\left(-L_{2}, 0\right)$ appears in both expressions in (4.12-4.13) and correspondingly for the reflection operator over the sub-slab $\left(-L,-L_{2}\right)$. Again such a pairing will lead to an expression for the corresponding cross correlation in terms of a statistically stable filter as we discuss below.

5. Characterization of cross correlations. Here, we characterize the "field-field" cross correlation function between the points $\left(-L_{1}, \varepsilon^{2} \boldsymbol{x}_{1}\right)$ and $\left(-L_{2}, \varepsilon^{2} \boldsymbol{x}_{2}\right)$. We assume that $T \gg L$ and $0<L_{1}<L_{2}<L$. The field-field correlation function is given by

$$
\mathcal{V}_{T}^{\varepsilon}(t)=\int_{0}^{T} p\left(t^{\prime},-L_{1}, \varepsilon^{2} \boldsymbol{x}_{1}\right) p\left(t^{\prime}+t,-L_{2}, \varepsilon^{2} \boldsymbol{x}_{2}\right) d t^{\prime} .
$$

Note that since we assume the source function $\chi$ to be real valued so is the pressure field. The configuration is illustrated in Figure 5.1a; we compute the correlation in between the two points at depth $-L_{1}$ and $-L_{2}$.

We will see that the wave field correlation function concentrates around specific time lags $t$ that correspond to travel times between the two observation points, and that the time extent of the correlation function around these time lags is of order $\varepsilon^{4}$, i.e., of the same order as the source pulse width.

Under the scaling assumptions of Section 2 we find that the correlations in (5.1) have leading 
contributions centered at four particular time lags:

$$
\begin{aligned}
& \mathcal{V}_{T}^{\varepsilon}\left( \pm\left(L_{2}-L_{1}\right)+\varepsilon^{4} s\right) / \varepsilon^{4} \simeq \mathcal{V}_{\mathrm{t}}^{ \pm}(s) \\
& \mathcal{V}_{T}^{\varepsilon}\left( \pm\left(2 L-L_{1}-L_{2}\right)+\varepsilon^{4} s\right) / \varepsilon^{4} \simeq \mathcal{V}_{\mathrm{r}}^{ \pm}(s)
\end{aligned}
$$

Here, the amplitude scaling corresponds to a rescaling of the source time traces so that they have order one energy, but plays no significant role as the problem is linear. The contributions $\mathcal{V}_{t}^{ \pm}$correspond to correlation of wave components directly transmitted in between the points of observation. The contribution around time lag equal to $+\left(L_{2}-L_{1}\right)$ comes from the correlation between the waves that propagate from the surface to the depth $-L_{1}$ and then to the depth $-L_{2}$ (see Figure 5.1b). The contribution around time lag equal to $-\left(L_{2}-L_{1}\right)$ comes from the correlation between the waves that have been reflected by the interface at $z=-L$ and that propagate from this interface to the depth $-L_{2}$ and then to the depth $-L_{1}$. We stress here that these wave components have been strongly affected by the multiple scattering in the medium and understanding how this affects the relation of the components $\mathcal{V}$ and the Green's functions is our main objective. The contribution $\mathcal{V}_{\mathrm{r}}^{ \pm}$corresponds to cross terms. The contribution around time lag equal to $2 L-L_{1}-L_{2}$ comes from correlation in between waves that have propagated from the surface to the reflector at depth $-L$ and then back to the depth $-L_{2}$ with the waves that have traveled directly to depth $-L_{1}$. Correspondingly the contribution around time lag equal to $-\left(2 L-L_{1}-L_{2}\right)$ comes from correlation in between waves that has propagated from the surface to the reflector at depth $-L$ and then back to the depth $-L_{1}$ with the waves that has traveled directly to depth $-L_{2}$. We remark here that the two last terms give contributions for larger time lags than the first two contributions (since $2 L-L_{1}-L_{2}>L_{2}-L_{1}$ ) and could be used for estimation of the depth of the bottom interface.

6. Integral expressions for correlation components. The correlation component $\mathcal{V}_{t}^{+}$can be characterized in distribution in the scaling limit $\varepsilon \rightarrow 0$ by the following expression using (4.10), (4.11) and the definition (5.1):

$$
\mathcal{V}_{\mathrm{t}}^{+}(s)=\frac{1}{2 \pi} \iint \check{\Lambda}_{\mathrm{t}}^{+}\left(\omega, \boldsymbol{x} ; \boldsymbol{x}_{1}, L_{1}\right) \check{\mathcal{T}}\left(\omega,-L_{2},-L_{1}, \boldsymbol{x}_{2}, \boldsymbol{x}_{1}-\boldsymbol{x}\right) d \boldsymbol{x} e^{-i \omega s} d \omega,
$$

where $\check{\mathcal{T}}\left(\omega,-L, z, \boldsymbol{x}, \boldsymbol{x}^{\prime}\right)$ is defined by (4.8). Remember that $\check{\mathcal{T}}\left(\omega,-L_{2},-L_{1}, \boldsymbol{x}_{2}, \boldsymbol{x}_{1}\right)$ is the (rescaled) paraxial Green's function from the point $\left(-L_{1}, \varepsilon^{2} \boldsymbol{x}_{1}\right)$ to the point $\left(-L_{2}, \varepsilon^{2} \boldsymbol{x}_{2}\right)$. Therefore, (6.1) reads as a filtered version of the causal Green's function in between the two points of observation. The filter is

$$
\check{\Lambda}_{\mathrm{t}}^{+}\left(\omega, \boldsymbol{x} ; \boldsymbol{x}_{1}, L_{1}\right)=\frac{1}{4} \iint \check{\mathcal{T}}\left(\omega,-L_{1}, 0, \boldsymbol{x}_{1}-\boldsymbol{x}, \boldsymbol{y}_{2}\right) \overline{\mathcal{T}}\left(\omega,-L_{1}, 0, \boldsymbol{x}_{1}, \boldsymbol{y}_{1}\right) \check{\chi}\left(\omega, \boldsymbol{y}_{2}\right) \overline{\check{\chi}\left(\omega, \boldsymbol{y}_{1}\right)} d \boldsymbol{y}_{1} d \boldsymbol{y}_{2} .
$$

In the regime we consider the filter $\Lambda_{\mathrm{t}}^{+}$defining the relevant transformation is self-averaging [8] in the sense that

$$
\begin{aligned}
& \check{\Lambda}_{\mathrm{t}}^{+}\left(\omega, \boldsymbol{x} ; \boldsymbol{x}_{1}, L_{1}\right)=\frac{1}{4} \iint \mathbb{E}\left[\check{\mathcal{T}}\left(\omega,-L_{1}, 0, \boldsymbol{x}_{1}-\boldsymbol{x}, \boldsymbol{y}_{2}\right) \overline{\mathcal{T}}\left(\omega,-L_{1}, 0, \boldsymbol{x}_{1}, \boldsymbol{y}_{1}\right)\right] \\
& \times \check{\chi}\left(\omega, \boldsymbol{y}_{2}\right) \bar{\chi}\left(\omega, \boldsymbol{y}_{1}\right) d \boldsymbol{y}_{1} d \boldsymbol{y}_{2},
\end{aligned}
$$

and we will study the properties of this filter in a regime of strong clutter in the next sections. Note that the fact that the filter is statistically stable is important from the theoretical point of view as it allows us to perform a detailed resolution analysis. It is important in practice because it guarantees that the quality of the Green's function estimation does not depend on the particular realization of the medium. It can also be used in order to improve the quality of the estimation: if the statistical distribution of the random medium is known, then the properties of the filter can be fully characterized (as is done in the next section) so that it is possible to (partially) deconvolve the effect of the filter and to enhance the estimation of the Green's function.

We have similarly, using the representations (4.12), (4.13):

$$
\mathcal{V}_{\mathrm{t}}^{-}(s)=\frac{\mathcal{R}_{0}^{2}}{2 \pi} \iint \check{\Lambda}_{\mathrm{t}}^{-}\left(\omega, \boldsymbol{x} ; \boldsymbol{x}_{2}, L_{2}, L\right) \check{\mathcal{T}}\left(\omega,-L_{1},-L_{2}, \boldsymbol{x}_{1}, \boldsymbol{x}_{2}-\boldsymbol{x}\right) d \boldsymbol{x} e^{-i \omega s} d \omega,
$$


with the filter

$$
\begin{aligned}
& \check{\Lambda}_{\mathrm{t}}^{-}\left(\omega, \boldsymbol{x} ; \boldsymbol{x}_{2}, L_{2}, L\right)=\frac{1}{4} \iint \check{\mathcal{T}}\left(\omega,-L_{2}, 0, \boldsymbol{y}_{4}, \boldsymbol{y}_{2}\right) \overline{\mathcal{T}}\left(\omega,-L_{2}, 0, \boldsymbol{y}_{3}, \boldsymbol{y}_{1}\right) \\
& \times \check{\mathcal{R}}\left(\omega,-L,-L_{2}, \boldsymbol{x}_{2}-\boldsymbol{x}, \boldsymbol{y}_{4}\right) \overline{\mathcal{R}}\left(\omega,-L,-L_{2}, \boldsymbol{x}_{2}, \boldsymbol{y}_{3}\right) \check{\chi}\left(\omega, \boldsymbol{y}_{2}\right) \overline{\check{\chi}\left(\omega, \boldsymbol{y}_{1}\right)} d \boldsymbol{y}_{1} d \boldsymbol{y}_{2} d \boldsymbol{y}_{3} d \boldsymbol{y}_{4} .
\end{aligned}
$$

Using reciprocity and the fact that $\mathcal{V}_{\mathrm{t}}^{-}(s)$ is real-valued, we have

$$
\mathcal{V}_{\mathrm{t}}^{-}(s)=\frac{\mathcal{R}_{0}^{2}}{2 \pi} \iint \check{\Lambda}_{\mathrm{t}}^{-}\left(\omega, \boldsymbol{x} ; \boldsymbol{x}_{2}, L_{2}, L\right) \check{\mathcal{T}}\left(\omega,-L_{2},-L_{1}, \boldsymbol{x}_{2}-\boldsymbol{x}, \boldsymbol{x}_{1}\right) d \boldsymbol{x} e^{i \omega s} d \omega,
$$

which shows that we obtain a filtered version of the anti-causal Green's function in between the two points of observation (note the sign in the exponent $e^{i \omega s}$ ). Self-averaging implies that

$$
\begin{aligned}
& \check{\Lambda}_{\mathrm{t}}^{-}\left(\omega, \boldsymbol{x} ; \boldsymbol{x}_{2}, L_{2}, L\right)=\frac{1}{4} \iint \mathbb{E}\left[\check{\mathcal{T}}\left(\omega,-L_{2}, 0, \boldsymbol{y}_{4}, \boldsymbol{y}_{2}\right) \overline{\mathcal{\mathcal { T }}}\left(\omega,-L_{2}, 0, \boldsymbol{y}_{3}, \boldsymbol{y}_{1}\right)\right] \\
& \quad \times \mathbb{E}\left[\check{\mathcal{R}}\left(\omega,-L,-L_{2}, \boldsymbol{x}_{2}-\boldsymbol{x}, \boldsymbol{y}_{4}\right) \overline{\mathcal{R}}\left(\omega,-L,-L_{2}, \boldsymbol{x}_{2}, \boldsymbol{y}_{3}\right)\right] \check{\chi}\left(\omega, \boldsymbol{y}_{2}\right) \overline{\check{\chi}\left(\omega, \boldsymbol{y}_{1}\right)} d \boldsymbol{y}_{1} d \boldsymbol{y}_{2} d \boldsymbol{y}_{3} d \boldsymbol{y}_{4} .
\end{aligned}
$$

Note that, the presence of the reflection operator in the expression for the filter (6.5) modifies the expression for the filter in a way that depends critically on the particular scaling regime considered. We discuss the explicit representation of the filter below.

For completeness we mention that the cross terms can be expressed in terms of the filter $\check{\Lambda}_{\mathrm{t}}^{+}$ defined by (6.2). We have

$$
\begin{aligned}
\mathcal{V}_{\mathrm{r}}^{+}(s)=\frac{\mathcal{R}_{0}}{2 \pi} \iint & \check{\Lambda}_{\mathrm{t}}^{+}\left(\omega, \boldsymbol{x} ; \boldsymbol{x}_{1}, L_{1}\right) \\
& \times\left\{\int \check{\mathcal{R}}\left(\omega,-L,-L_{2}, \boldsymbol{x}_{2}, \boldsymbol{y}\right) \check{\mathcal{T}}\left(\omega,-L_{2},-L_{1}, \boldsymbol{y}, \boldsymbol{x}_{1}-\boldsymbol{x}\right) d \boldsymbol{y}\right\} d \boldsymbol{x} e^{-i \omega s} d \omega,
\end{aligned}
$$

and

$$
\begin{aligned}
\mathcal{V}_{\mathrm{r}}^{-}(s)=\frac{\mathcal{R}_{0}}{2 \pi} \iint & \check{\Lambda}_{\mathrm{t}}^{+}\left(\omega, \boldsymbol{x} ; \boldsymbol{x}_{2}, L_{2}\right) \\
& \times\left\{\int \check{\mathcal{T}}\left(\omega,-L_{1},-L_{2}, \boldsymbol{x}_{1}, \boldsymbol{y}\right) \check{\mathcal{R}}\left(\omega,-L,-L_{2}, \boldsymbol{y}, \boldsymbol{x}_{2}-\boldsymbol{x}\right) d \boldsymbol{y}\right\} d \boldsymbol{x} e^{-i \omega s} d \omega .
\end{aligned}
$$

7. Filters via Wigner transforms. In order to characterize the filter $\Lambda_{t}^{+}$we introduce the Wigner transform of the transmission operator defined by

$$
\begin{aligned}
& W_{\omega}^{\mathrm{T}}\left(z, \boldsymbol{x}, \boldsymbol{x}^{\prime}, \boldsymbol{\kappa}, \boldsymbol{\kappa}^{\prime}\right)= \\
& \quad \iint e^{-i\left(\boldsymbol{\kappa} \cdot \boldsymbol{y}+\boldsymbol{\kappa}^{\prime} \cdot \boldsymbol{y}^{\prime}\right)} \mathbb{E}\left[\check{\mathcal{T}}\left(\omega,-z, 0, \boldsymbol{x}+\frac{\boldsymbol{y}}{2}, \boldsymbol{x}^{\prime}+\frac{\boldsymbol{y}^{\prime}}{2}\right) \overline{\check{\mathcal{T}}\left(\omega,-z, 0, \boldsymbol{x}-\frac{\boldsymbol{y}}{2}, \boldsymbol{x}^{\prime}-\frac{\boldsymbol{y}^{\prime}}{2}\right)}\right] d \boldsymbol{y} d \boldsymbol{y}^{\prime} .
\end{aligned}
$$

The Wigner transforms satisfy a set of transport equations:

$$
\frac{\partial W_{\omega}^{\mathrm{T}}}{\partial z}+\frac{\boldsymbol{\kappa}^{\prime}}{\omega} \nabla_{\boldsymbol{x}^{\prime}} W_{\omega}^{\mathrm{T}}=\frac{\omega^{2}}{4} \frac{1}{(2 \pi)^{d}} \int \hat{D}(\boldsymbol{u})\left[W_{\omega}^{\mathrm{T}}\left(z, \boldsymbol{x}, \boldsymbol{x}^{\prime}, \boldsymbol{\kappa}, \boldsymbol{\kappa}^{\prime}-\boldsymbol{u}\right)-W_{\omega}^{\mathrm{T}}\left(z, \boldsymbol{x}, \boldsymbol{x}^{\prime}, \boldsymbol{\kappa}, \boldsymbol{\kappa}^{\prime}\right)\right] d \boldsymbol{u},
$$

starting from $W_{\omega}^{\mathrm{T}}\left(z=0, \boldsymbol{x}, \boldsymbol{x}^{\prime}, \boldsymbol{\kappa}, \boldsymbol{\kappa}^{\prime}\right)=(2 \pi)^{d} \delta\left(\boldsymbol{x}-\boldsymbol{x}^{\prime}\right) \delta\left(\boldsymbol{\kappa}+\boldsymbol{\kappa}^{\prime}\right)$. This system can be integrated [7] and we find the following integral representation for $W_{\omega}^{\mathrm{T}}$ :

$$
W_{\omega}^{\mathrm{T}}\left(z, \boldsymbol{x}, \boldsymbol{x}^{\prime}, \boldsymbol{\kappa}, \boldsymbol{\kappa}^{\prime}\right)=\frac{1}{(2 \pi)^{d}} \iint e^{-i\left(\boldsymbol{\kappa}^{\prime}+\boldsymbol{\kappa}\right) \cdot \boldsymbol{a}-i\left(\boldsymbol{x}^{\prime}-\boldsymbol{x}+\frac{\kappa}{\omega} z\right) \cdot \boldsymbol{b}} e^{\frac{\omega^{2}}{4} \int_{0}^{z} D\left(\boldsymbol{a}+\frac{b}{\omega} z^{\prime}\right)-D(\mathbf{0}) d z^{\prime}} d \boldsymbol{a} d \boldsymbol{b} .
$$

In order to characterize the filter $\Lambda_{\mathrm{t}}^{-}$we also introduce the Wigner transform of the reflection operator defined by

$$
\begin{aligned}
& W_{\omega}^{\mathrm{R}}\left(z, \boldsymbol{x}, \boldsymbol{x}^{\prime}, \boldsymbol{\kappa}, \boldsymbol{\kappa}^{\prime}\right)= \\
& \quad \iint e^{-i\left(\boldsymbol{\kappa} \cdot \boldsymbol{y}+\boldsymbol{\kappa}^{\prime} \cdot \boldsymbol{y}^{\prime}\right)} \mathbb{E}\left[\check{\mathcal{R}}\left(\omega,-L,-z, \boldsymbol{x}+\frac{\boldsymbol{y}}{2}, \boldsymbol{x}^{\prime}+\frac{\boldsymbol{y}^{\prime}}{2}\right) \overline{\mathcal{R}\left(\omega,-L,-z, \boldsymbol{x}-\frac{\boldsymbol{y}}{2}, \boldsymbol{x}^{\prime}-\frac{\boldsymbol{y}^{\prime}}{2}\right)}\right] d \boldsymbol{y} d \boldsymbol{y}^{\prime} .
\end{aligned}
$$


It satisfies a set of transport equations:

$$
\begin{aligned}
& \frac{\partial W_{\omega}^{\mathrm{R}}}{\partial z}+\frac{\boldsymbol{\kappa}}{\omega} \cdot \nabla_{\boldsymbol{x}} W_{\omega}^{\mathrm{R}}+\frac{\boldsymbol{\kappa}^{\prime}}{\omega} \cdot \nabla_{\boldsymbol{x}^{\prime}} W_{\omega}^{\mathrm{R}}=\frac{\omega^{2}}{4(2 \pi)^{d}} \int \hat{D}(\boldsymbol{u}) \\
& \times \quad\left[W_{\omega}^{\mathrm{R}}\left(z, \boldsymbol{x}, \boldsymbol{x}^{\prime}, \boldsymbol{\kappa}-\boldsymbol{u}, \boldsymbol{\kappa}^{\prime}\right)+W_{\omega}^{\mathrm{R}}\left(z, \boldsymbol{x}, \boldsymbol{x}^{\prime}, \boldsymbol{\kappa}, \boldsymbol{\kappa}^{\prime}-\boldsymbol{u}\right)\right. \\
& \quad+2 W_{\omega}^{\mathrm{R}}\left(z, \boldsymbol{x}, \boldsymbol{x}^{\prime}, \boldsymbol{\kappa}-\frac{1}{2} \boldsymbol{u}, \boldsymbol{\kappa}^{\prime}-\frac{1}{2} \boldsymbol{u}\right) \cos \left(\boldsymbol{u} \cdot\left(\boldsymbol{x}-\boldsymbol{x}^{\prime}\right)\right) \\
& \quad-2 W_{\omega}^{\mathrm{R}}\left(z, \boldsymbol{x}, \boldsymbol{x}^{\prime}, \boldsymbol{\kappa}-\frac{1}{2} \boldsymbol{u}, \boldsymbol{\kappa}^{\prime}+\frac{1}{2} \boldsymbol{u}\right) \cos \left(\boldsymbol{u} \cdot\left(\boldsymbol{x}-\boldsymbol{x}^{\prime}\right)\right) \\
& \left.\quad-2 W_{\omega}^{\mathrm{R}}\left(z, \boldsymbol{x}, \boldsymbol{x}^{\prime}, \boldsymbol{\kappa}, \boldsymbol{\kappa}^{\prime}\right)\right] d \boldsymbol{u},
\end{aligned}
$$

starting from $W_{\omega}^{\mathrm{R}}\left(z=-L, \boldsymbol{x}, \boldsymbol{x}^{\prime}, \boldsymbol{\kappa}, \boldsymbol{\kappa}^{\prime}\right)=(2 \pi)^{d} \delta\left(\boldsymbol{x}-\boldsymbol{x}^{\prime}\right) \delta\left(\boldsymbol{\kappa}+\boldsymbol{\kappa}^{\prime}\right)$.

The filters can now be expressed as

$$
\begin{aligned}
& \check{\Lambda}_{\mathrm{t}}^{+}\left(\omega, \boldsymbol{x} ; \boldsymbol{x}_{1}, L_{1}\right)=\frac{1}{4(2 \pi)^{2 d}} \iint e^{-i\left(\boldsymbol{\kappa} \cdot \boldsymbol{x}+\boldsymbol{\kappa}^{\prime} \cdot\left(\boldsymbol{y}_{1}-\boldsymbol{y}_{2}\right)\right)} W_{\omega}^{\mathrm{T}}\left(L_{1}, \boldsymbol{x}_{1}-\boldsymbol{x} / 2,\left(\boldsymbol{y}_{1}+\boldsymbol{y}_{2}\right) / 2, \boldsymbol{\kappa}, \boldsymbol{\kappa}^{\prime}\right) d \boldsymbol{\kappa} d \boldsymbol{\kappa}^{\prime} \\
& \times \check{\chi}\left(\omega, \boldsymbol{y}_{2}\right) \bar{\chi}\left(\omega, \boldsymbol{y}_{1}\right) d \boldsymbol{y}_{1} d \boldsymbol{y}_{2}, \\
& \check{\Lambda}_{\mathrm{t}}^{-}\left(\omega, \boldsymbol{x} ; \boldsymbol{x}_{2}, L_{2}\right)=\frac{1}{4(2 \pi)^{4 d}} \iint e^{i\left(\boldsymbol{\kappa} \cdot\left(\boldsymbol{y}_{4}-\boldsymbol{y}_{3}\right)+\boldsymbol{\kappa}^{\prime} \cdot\left(\boldsymbol{y}_{2}-\boldsymbol{y}_{1}\right)\right)} W_{\omega}^{\mathrm{T}}\left(L_{2},\left(\boldsymbol{y}_{3}+\boldsymbol{y}_{4}\right) / 2,\left(\boldsymbol{y}_{1}+\boldsymbol{y}_{2}\right) / 2, \boldsymbol{\kappa}, \boldsymbol{\kappa}^{\prime}\right) d \boldsymbol{\kappa} d \boldsymbol{\kappa}^{\prime}
\end{aligned}
$$

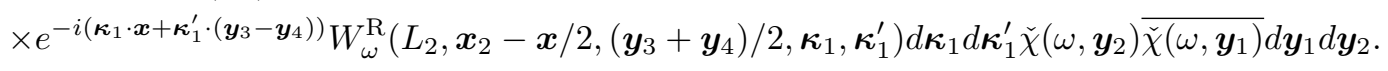

Here, $W_{\omega}^{\mathrm{T}}$ is given by the integral expression in (7.1) and we shall discuss below specific scaling regimes that give explicit expressions for both $W_{\omega}^{\mathrm{T}}$ and $W_{\omega}^{\mathrm{R}}$. Indeed, in order to get explicit forms for the filters and characterize the associated resolution scales, or filter support scales, we consider next a particular regime of relatively strong clutter.

8. The strongly cluttered scaling regime. We introduce next the correlation length, $l$, of the random medium fluctuations, $\omega_{c}$ the characteristic relative frequency of the source time trace and the lateral cross section $r_{0}$ of the source:

$$
\begin{aligned}
& C(z, \boldsymbol{x})=\sigma^{2} C_{0}\left(\frac{z}{l}, \frac{\boldsymbol{x}}{l}\right), \\
& \check{\chi}(\omega, \boldsymbol{y})=\check{\chi}_{0}\left(\frac{\omega}{\omega_{c}}, \frac{\boldsymbol{y}}{r_{0}}\right),
\end{aligned}
$$

where $\sigma$ is the standard deviation of the fluctuations of the random medium. We assume that $\check{\chi}_{0}$ is supported away from the origin in frequency.

With this representation we have

$$
D(\boldsymbol{x})=\sigma^{2} l D_{0}\left(\frac{\boldsymbol{x}}{l}\right)
$$

We assume also that the autocorrelation function $D_{0}(\boldsymbol{x})$ is at least twice differentiable at $\boldsymbol{x}=\mathbf{0}$, which corresponds to a smooth random medium. We introduce then the parameter $\gamma$ defined by:

$$
\gamma=\frac{1}{d} \frac{1}{(2 \pi)^{d}} \int|\boldsymbol{u}|^{2} \hat{D}(\boldsymbol{u}) d \boldsymbol{u}=-\frac{1}{d} \Delta D(\mathbf{0})=-\frac{\sigma^{2}}{d l} \Delta D_{0}(\mathbf{0})=\frac{\sigma^{2}}{l} \gamma_{0}, \quad \gamma_{0}=-\frac{1}{d} \Delta D_{0}(\mathbf{0}) .
$$

We introduce the parameter, depending on $(\omega, z)$,

$$
\beta(\omega, z)=z \frac{\sigma^{2} \omega^{2} l}{4},
$$

which characterizes the strength of the forward scattering. We shall then assume a subsequent scaling regime corresponding to relatively strong medium interaction: 
Assumption 2. $\beta\left(\omega_{c}, L_{1}\right) \gg 1$.

This scaling may for instance be generated by $\sigma \gg 1$, that is, relatively strong clutter fluctuations. We remark that this entails for $\omega \sim \omega_{c}, z \sim L_{1}$ :

$$
\frac{1}{\sqrt{\beta(\omega, z)}}\left(\frac{l}{2 \sqrt{\gamma_{0}}}\right)=\frac{1}{\sqrt{\gamma \omega^{2} z}} \ll r_{0} \ll \sqrt{\gamma z^{3}}=\sqrt{\beta(\omega, z)}\left(\frac{2 z \sqrt{\gamma_{0}}}{\omega l}\right) .
$$

We next introduce the lateral correlation scale of the transmitted wave field, $\rho_{\mathrm{T}}(z, \omega)$, and the support scale of the forward cone of wave energy, $r_{\mathrm{T}}(z)[6,8]$ :

$$
\rho_{\mathrm{T}}(z, \omega)=\frac{4}{\sqrt{\gamma \omega^{2} z}}, \quad r_{\mathrm{T}}(z)=\sqrt{\frac{\gamma z^{3}}{3}} .
$$

We will see below that these two quantities determine the behavior of the filter in (6.2). By (8.2) we have

$$
\rho_{\mathrm{T}}(z, \omega) \ll r_{0} \ll r_{\mathrm{T}}(z) .
$$

We summarize the scaling assumptions. The main assumption that $\varepsilon \ll 1$ corresponds to very rapid and small medium fluctuations relative to the propagation distance and a high frequency parabolic source scaling. To get explicit representations for the integral expressions defining the filter $\Lambda_{\mathrm{t}}^{+}$we assume a strong clutter interaction corresponding to strong forward scattering in Section 9.

9. Filter asymptotics for the causal Green's function estimate. In Appendix A we derive the following asymptotic expression for $\check{\Lambda}_{\mathrm{t}}^{+}$that is valid in the strongly cluttered regime.

Proposition 9.1. Under Assumption 2, the filter $\check{\Lambda}_{\mathrm{t}}^{+}$defined by (6.2) has the following representation for $0<z<L$

$$
\begin{aligned}
\check{\Lambda}_{\mathrm{t}}^{+}\left(\omega, \boldsymbol{x} ; \boldsymbol{x}_{1}, z\right) \simeq & \frac{1}{4}\left(\frac{6}{\pi \gamma z^{3}}\right)^{d / 2} \exp \left(-\frac{\gamma \omega^{2} z}{32}|\boldsymbol{x}|^{2}\right) \exp \left(-\frac{6}{\gamma z^{3}}\left|\boldsymbol{x}_{1}\right|^{2}\right) \\
& \times e^{-i \frac{3 \omega}{2 z} \boldsymbol{x}_{1} \cdot \boldsymbol{x}} \int|\check{\chi}(\omega, \boldsymbol{y})|^{2} d \boldsymbol{y} \\
= & \frac{1}{4}\left(\frac{2}{\pi r_{\mathrm{T}}^{2}(z)}\right)^{d / 2} \exp \left(-\frac{|\boldsymbol{x}|^{2}}{2 \rho_{\mathrm{T}}^{2}(z, \omega)}\right) \exp \left(-\frac{2\left|\boldsymbol{x}_{1}\right|^{2}}{r_{\mathrm{T}}^{2}(z)}\right) \\
& \times e^{-i \sqrt{12} \frac{\boldsymbol{x}}{\rho_{\mathrm{T}}(z, \omega)} \cdot \frac{\boldsymbol{x}_{1}}{r_{\mathrm{T}}(z)}} \int|\check{\chi}(\omega, \boldsymbol{y})|^{2} d \boldsymbol{y} .
\end{aligned}
$$

The filter $\Lambda_{t}^{+}$governs the resolution with which the correlation component $\mathcal{V}_{t}^{+}$resolves the corresponding empirical Green's function as described in (6.1), that is, the causal Green's function. The support of the filter $\Lambda_{\mathrm{t}}^{+}$in $\boldsymbol{x}$ determines the resolution in the estimate $\mathcal{V}_{\mathrm{t}}^{+}$of the Green's function, or the amount of blurring in the source coordinate. This is determined by the lateral correlation length $\rho_{\mathrm{T}}\left(L_{1}, \omega\right)$, the lateral coherence scale of the wave field at depth $-L_{1}$. It also can be seen from (9.1) that the source point $\boldsymbol{x}_{1}$ must be in the forward cone of wave energy, as determined by $r_{\mathrm{T}}(z)$, in order to enable Green's function estimation. We stress here that a relatively sharp Green's function estimate is enabled by a small $\rho_{T}$ and an enhanced width of the forward cone, both induced by strong medium clutter.

The Green's function estimate by $\mathcal{V}_{t}^{+}$will also be blurred in time according to the bandwidth of the source trace. We have using (6.1) and (9.1)

$$
\mathcal{V}_{\mathrm{t}}^{+}(s)=\iint \Lambda_{\mathrm{t}}^{+}\left(t, \boldsymbol{x} ; \boldsymbol{x}_{1}, L_{1}\right) \mathcal{T}\left(s-t,-L_{2},-L_{1}, \boldsymbol{x}_{2}, \boldsymbol{x}_{1}-\boldsymbol{x}\right) d \boldsymbol{x} d t,
$$

for

$$
\begin{aligned}
\Lambda_{\mathrm{t}}^{+}\left(t, \boldsymbol{x} ; \boldsymbol{x}_{1}, z\right)= & \frac{1}{4}\left(\frac{2}{\pi r_{\mathrm{T}}^{2}(z)}\right)^{d / 2} \exp \left(-\frac{2\left|\boldsymbol{x}_{1}\right|^{2}}{r_{\mathrm{T}}^{2}(z)}\right) \\
& \times \frac{\tilde{\rho}_{\mathrm{T}}(z)}{\sqrt{2 \pi}|\boldsymbol{x}|} \int \exp \left(-\frac{\tilde{\rho}_{\mathrm{T}}^{2}(z) s^{2}}{2|\boldsymbol{x}|^{2}}\right) \Phi\left(t+\theta_{\mathrm{T}}\left(\boldsymbol{x}, \boldsymbol{x}_{1}, z\right)-s\right) d s .
\end{aligned}
$$


Here, we used the notations:

$$
\Phi(s)=\iint \chi(t, \boldsymbol{y}) \chi(t+s, \boldsymbol{y}) d \boldsymbol{y} d t, \quad \tilde{\rho}_{\mathrm{T}}(z)=\frac{4}{\sqrt{\gamma z}}, \quad \theta_{\mathrm{T}}\left(\boldsymbol{x}, \boldsymbol{x}_{1}, z\right)=\sqrt{12} \frac{\boldsymbol{x}}{\tilde{\rho}_{\mathrm{T}}(z)} \cdot \frac{\boldsymbol{x}_{1}}{r_{\mathrm{T}}(z)} .
$$

Thus, the cross correlation gives a blurred Green's function, corresponding to smoothing in time and source point and with a slight time shift for contributions with lateral offsets. The spatial source point focusing follows from the increase of support in the time smoothing for lateral offset.

In the case when $\chi$ has a narrow bandwidth and carrier frequency $\omega_{c}$, we have

$$
\Lambda_{\mathrm{t}}^{+}\left(t, \boldsymbol{x} ; \boldsymbol{x}_{1}, z\right)=\frac{1}{4}\left(\frac{2}{\pi r_{\mathrm{T}}^{2}(z)}\right)^{d / 2} \exp \left(-\frac{|\boldsymbol{x}|^{2}}{2 \rho_{\mathrm{T}}^{2}\left(z, \omega_{c}\right)}\right) \exp \left(-\frac{2\left|\boldsymbol{x}_{1}\right|^{2}}{r_{\mathrm{T}}^{2}(z)}\right) \Phi\left(t+\theta_{\mathrm{T}}\left(\boldsymbol{x}, \boldsymbol{x}_{1}, z\right)\right) .
$$

Finally, if we also assume a relatively small offset, $\left|\boldsymbol{x}_{1}\right| \ll r_{\mathrm{T}}\left(L_{1}\right)$, we have

$$
\Lambda_{\mathrm{t}}^{+}\left(t, \boldsymbol{x} ; \boldsymbol{x}_{1}, L_{1}\right)=\frac{1}{4}\left(\frac{2}{\pi r_{\mathrm{T}}^{2}\left(L_{1}\right)}\right)^{d / 2} \exp \left(-\frac{|\boldsymbol{x}|^{2}}{2 \rho_{\mathrm{T}}^{2}\left(L_{1}, \omega_{c}\right)}\right) \Phi(t) .
$$

In conclusion, the Green's function in (9.2) is blurred in time according to the autocorrelation of the source time trace and in space according to the Gaussian with support determined by the spatial decorrelation length of the wave field at $z=-L_{1}$ when emanating from a point source at $z=0$ and with carrier $\omega_{c}$. There is also a damping of the filter with the widening of the forward cone. We recall that this final description holds under the assumption that $\boldsymbol{x}_{1}$ is within the forward cone of wave energy, or $\left|\boldsymbol{x}_{1}\right|<r_{\mathrm{T}}\left(L_{1}\right)$.

10. Filter asymptotics for the anticausal Green's function estimate. We now turn to the filter $\Lambda_{\mathrm{t}}^{-}$that gives the anticausal contribution in (6.3). We shall here consider the regime of rapid clutter. In this case we assume

Assumption 3. $r_{0} \gg l, \quad \omega_{c} r_{0} l=\mathcal{O}(L)$.

We remark that in the random medium case diffractive effects are of order one when $\omega_{c} r_{0} l \sim L$. This is when the Rayleigh length associated with the Fresnel length, $\sqrt{r_{0} l}$, is of order the depth of the slab. Note that in this configuration the random medium fluctuations give an earlier onset of diffractive effects than in the homogeneous case.

In this case we show in Appendix B the following asymptotic representation for the filter $\check{\Lambda}_{t}$ that is valied in the strongly cluttered regime.

Proposition 10.1. Under Assumptions 2-3, the filter $\check{\Lambda}_{\mathrm{t}}^{-}$defined by (6.5) has the representation

$$
\check{\Lambda}_{t}^{-}\left(\omega, \boldsymbol{x} ; \boldsymbol{x}_{1}, z, L\right) \simeq \check{\Lambda}_{t}^{+}\left(\omega, \boldsymbol{x} ; \boldsymbol{x}_{1}, 2 L-z\right) .
$$

Therefore, the spatial resolution for the filter with a relatively deep reflector (ie. $L \gg z$ ) is as if the field propagated in a medium corresponding to twice the travel distance to the reflector at $-L$ and acquires the corresponding shortened spatial decorrelation length. Note that with a deep reflector, with $L$ much larger than $L_{1}$, the filter $\Lambda_{t}^{+}$gives much better resolution than the filter $\Lambda_{t}^{-}$, at the cost of a reduced amplitude. In this case the cross correlation presents a strong asymmetry between the causal and anti-causal components.

In the special case that $L \simeq z$ we have

$$
\Lambda_{\mathrm{t}}^{-}\left(t, \boldsymbol{x} ; \boldsymbol{x}_{1}, z, L\right) \simeq \Lambda_{\mathrm{t}}^{+}\left(t, \boldsymbol{x} ; \boldsymbol{x}_{1}, L\right),
$$

so that the filters coincide if the interface is located just below both of the measurements. In this case the cross correlation presents a symmetry between the causal and anti-causal components.

11. Horizontal moveout case. We consider here the situation when both the measurements are taken at the surface $z=0$. In this case the observations can be derived from (6.3):

$$
\begin{aligned}
\mathcal{V}_{\mathrm{t}}^{-}(s) & =\frac{\mathcal{R}_{0}^{2}}{2 \pi} \iint \check{\Lambda}_{\mathrm{t}}^{-}\left(\omega, \boldsymbol{x} ; \boldsymbol{x}_{2}, 0, L\right) \check{\mathcal{T}}\left(\omega, 0,0, \boldsymbol{x}_{1}, \boldsymbol{x}_{2}-\boldsymbol{x}\right) d \boldsymbol{x} e^{-i \omega s} d \omega \\
& =\mathcal{R}_{0}^{2} \Lambda_{\mathrm{t}}^{-}\left(s, \boldsymbol{x}_{2}-\boldsymbol{x}_{1} ; \boldsymbol{x}_{2}, 0, L\right) .
\end{aligned}
$$


Therefore, by varying the horizontal offset for the two measurement points we can estimate the filter, as a function of midpoint, offset (or $\boldsymbol{x}_{1}, \boldsymbol{x}_{2}$ ) and time. In the scaling limit that we consider the filter function gives a concise parameterization for the effective parameters associated with the medium that we can estimate. Under Assumptions 1, 2, 3, and assuming a narrow bandwidth for $\chi_{0}$, we have using (10.1) and (9.4)

$$
\begin{aligned}
& \check{\Lambda}_{\mathrm{t}}^{-}\left(s, \boldsymbol{x}_{2}-\boldsymbol{x}_{1} ; \boldsymbol{x}_{2}, 0, L\right) \\
& \quad \simeq \frac{1}{4}\left(\frac{3}{4 \pi \gamma L^{3}}\right)^{d / 2} \exp \left(-\frac{\gamma \omega_{c}^{2} L}{16}\left|\boldsymbol{x}_{1}-\boldsymbol{x}_{2}\right|^{2}\right) \exp \left(-\frac{3}{4 \gamma L^{3}}\left|\boldsymbol{x}_{2}\right|^{2}\right) \Phi\left(s+\theta_{\mathrm{R}}\left(\boldsymbol{x}_{2}-\boldsymbol{x}_{1}, \boldsymbol{x}_{2}, L\right)\right) \\
& \quad=\frac{1}{4}\left(\frac{2}{\pi r_{\mathrm{R}}^{2}(L)}\right)^{d / 2} \exp \left(-\frac{\left|\boldsymbol{x}_{1}-\boldsymbol{x}_{2}\right|^{2}}{2 \rho_{\mathrm{R}}^{2}\left(L, \omega_{c}\right)}\right) \exp \left(-\frac{2\left|\boldsymbol{x}_{2}\right|^{2}}{r_{\mathrm{R}}^{2}(L)}\right) \Phi\left(s+\theta_{\mathrm{R}}\left(\boldsymbol{x}_{2}-\boldsymbol{x}_{1}, \boldsymbol{x}_{2}, L\right)\right) \\
& \quad \simeq \frac{1}{4}\left(\frac{2}{\pi r_{\mathrm{R}}^{2}(L)}\right)^{d / 2} \exp \left(-\frac{\left|\boldsymbol{x}_{\text {off }}\right|^{2}}{2 \rho_{\mathrm{R}}^{2}\left(L, \omega_{c}\right)}\right) \exp \left(-\frac{2\left|\boldsymbol{x}_{\text {mid }}\right|^{2}}{r_{\mathrm{R}}^{2}(L)}\right) \Phi\left(s+\theta_{\mathrm{R}}\left(-\boldsymbol{x}_{\text {off }}, \boldsymbol{x}_{\text {mid }}, L\right)\right),
\end{aligned}
$$

where we used the notation

$$
\boldsymbol{x}_{\mathrm{mid}}=\frac{\boldsymbol{x}_{1}+\boldsymbol{x}_{2}}{2}, \quad \boldsymbol{x}_{\mathrm{off}}=\boldsymbol{x}_{1}-\boldsymbol{x}_{2}
$$

and

$$
\rho_{\mathrm{R}}\left(L, \omega_{c}\right)=\frac{4}{\sqrt{2 \omega_{c}^{2} \gamma L}}, \quad r_{\mathrm{R}}(z)=\sqrt{\frac{8 \gamma L^{3}}{3}} .
$$

Finally, we also have here

$$
\theta_{\mathrm{R}}\left(\boldsymbol{x}, \boldsymbol{x}_{1}, L\right)=\sqrt{12} \frac{\boldsymbol{x}}{\tilde{\rho}_{\mathrm{R}}(L)} \cdot \frac{\boldsymbol{x}_{1}}{r_{\mathrm{R}}(L)}, \quad \tilde{\rho}_{\mathrm{R}}(L)=\frac{4}{\sqrt{2 \gamma L}} .
$$

In the case that we have measurements along a line through the center of the source (the origin in our coordinate system) we can then observe, using various offsets and centerpoints, two parameters that encapsulate the "measurable" medium information:

$$
\mathcal{P}_{1}=\gamma L, \quad \mathcal{P}_{2}=\gamma L^{3} .
$$

The point is that measuring both the wave field decorrelation length and the energy cone spreading we can construct an estimate for depth without prior information about the medium microstructure. This is exactly the information the cross correlations convey in the regime that we consider. We stress that in a situation with finite scales the filter observations will not be perfectly stable with respect to the statistical distribution of the random medium. The observations at many offsets, centerpoints and possibly also carrier frequencies will be needed to get robust estimates of these parameters. The important point now is that we can obtain an estimate of the depth to the reflector and the medium correlation parameter from estimates $\hat{\mathcal{P}}_{1}$ and $\hat{\mathcal{P}}_{2}$

$$
\hat{L}=\sqrt{\frac{\hat{\mathcal{P}}_{2}}{\hat{\mathcal{P}}_{1}}}, \quad \hat{\gamma}=\sqrt{\frac{\hat{\mathcal{P}}_{1}^{3}}{\hat{\mathcal{P}}_{2}}} .
$$

The construction of the estimates $\hat{\mathcal{P}}_{1}, \hat{\mathcal{P}}_{2}$ need to be tuned to the particular configuration at hand. However, our scaling limit analysis gives guidelines for optimal design of measurement configuration for constructing such estimates. We also remark that this is just the starting point for a more elaborate analysis for cases where the medium background varies smoothly so that the medium fluctuations are locally stationary. Note that if we assume a general smooth background, then in our parabolic scaling we will have smooth order one variations with respect to depth, but slow variations with respect the lateral coordinates. Thus, this is a perturbation of the case with depth-dependent background only. If we consider next two measurements with large offset relative to the center point and possibly also large offset in between them, then in fact the effective medium parameters along 
the "rays" in between the source point and the points of observation can be estimated. This is a set-up for estimating fabric as well as deterministic discontinuities. Let us consider first the situation addressed above, in which the background velocity is constant. Then in the process of estimating the reflector depth function, $L(\boldsymbol{x})$, we also need to estimate the location-dependent correlation parameter map of the medium in the overburden, $\gamma(z, \boldsymbol{x})$. We stress that this correlation map is of importance as it may relate to the geological fabric. Note that if in addition the background velocity varies smoothly, then this estimation needs to be carried out jointly with a "velocity analysis". Our paper presents the first step towards imaging involving the joint estimate of the microstructure correlation function and the local velocity function based on both correlations and travel time estimates. Our method is particularly effective in the case with relatively strong medium and measurement noise: the recorded signals are then cluttered and formation of cross correlations is needed to stabilize the data.

12. Discussion. We presented an analysis for partly coherent body waves generated by a (teleseismic) wave packet remotely incident on a slab (the crust) containing a medium consisting of a deterministic component and a random field. We assume a parabolic scaling of the incident wave packet which is coupled to the scaling of the random fluctuations. In practical situations, such an incident wave packet may be synthesized from given observed data. The deterministic component consists, here, of a planarly layered medium, but can be generalized to contain conormal singularities (discontinuities) combined with smooth wave speed variations. To obtain information about the deterministic medium component, one needs to consider "field-field" cross correlations. We showed that these cross correlations are characterized by a statistically stable transformation (blurring filter) applied to the transmission operator given by an Itô-Schrödinger diffusion model which we interpret as the Green's function between two points. The blurring transformation contains information about the statistics of the random fluctuations. If the points are taken purely transverse to the propagation direction of the wave packet in the deterministic component, the blurring significantly increases, which is consistent with the usual stationary phase arguments. In principle, incident packets also can be summed to form a point source, to represent local seismicity.

Acknowledgments. The work was partially supported by NSF ARRA grant DMS 0908274 .

Appendix A. Correlation filter in strongly cluttered narrow bandwidth regime. In this appendix we derive the expression (9.1) for the filter. To get an explicit expression of the filter $\Lambda_{\mathrm{t}}^{+}$we need an expression for $W_{\omega}^{\mathrm{T}}$ in the strongly cluttered regime. With assumption 2 we obtain

$$
W_{\omega}^{\mathrm{T}}\left(z, \boldsymbol{x}, \boldsymbol{x}^{\prime}, \boldsymbol{\kappa}, \boldsymbol{\kappa}^{\prime}\right) \simeq \frac{1}{(2 \pi)^{d}} \iint e^{-i\left(\boldsymbol{\kappa}^{\prime}+\boldsymbol{\kappa}\right) \cdot \boldsymbol{a}-i\left(\boldsymbol{x}^{\prime}-\boldsymbol{x}+\frac{\boldsymbol{\kappa}}{\omega} z\right) \cdot \boldsymbol{b}} e^{-\frac{\gamma \omega^{2}}{8}\left(|\boldsymbol{a}|^{2} z+\frac{\boldsymbol{a} \cdot \boldsymbol{b}}{\omega} z^{2}+\frac{1}{3} \frac{|\boldsymbol{b}|^{2}}{\omega^{2}} z^{3}\right)} d \boldsymbol{a} d \boldsymbol{b} .
$$

Taking an inverse Fourier transform, we find that the cross correlation function of the kernel of transmission operator is

$$
\begin{aligned}
& \mathbb{E}\left[\check{\mathcal{T}}\left(\omega,-z, 0, \boldsymbol{x}+\frac{\boldsymbol{y}}{2}, \boldsymbol{x}^{\prime}+\frac{\boldsymbol{y}^{\prime}}{2}\right) \overline{\left.\check{\mathcal{T}}\left(\omega,-z, 0, \boldsymbol{x}-\frac{\boldsymbol{y}}{2}, \boldsymbol{x}^{\prime}-\frac{\boldsymbol{y}^{\prime}}{2}\right)\right]}\right. \\
& \quad \simeq\left(\frac{\omega}{2 \pi z}\right)^{d} e^{i \frac{\omega}{z}\left(\boldsymbol{x}^{\prime}-\boldsymbol{x}\right) \cdot\left(\boldsymbol{y}^{\prime}-\boldsymbol{y}\right)} e^{-\frac{\gamma \omega^{2} z}{8}\left(\frac{1}{3}\left|\boldsymbol{y}^{\prime}+\frac{1}{2} \boldsymbol{y}\right|^{2}+\frac{1}{4}|\boldsymbol{y}|^{2}\right)} .
\end{aligned}
$$

Using (6.2) and (A.2) we find:

$$
\begin{aligned}
& \check{\Lambda}_{\mathrm{t}}^{+}\left(\omega, \boldsymbol{x} ; \boldsymbol{x}_{1}, z\right)=\frac{1}{4} \iint\left(\frac{\omega}{2 \pi z}\right)^{d} e^{i \frac{\omega}{2 z}\left(\boldsymbol{y}_{1}+\boldsymbol{y}_{1}^{\prime}+\boldsymbol{x}-2 \boldsymbol{x}_{1}\right) \cdot\left(\boldsymbol{y}_{1}-\boldsymbol{y}_{1}^{\prime}+\boldsymbol{x}\right)} e^{-\frac{\gamma \omega^{2} z}{8}\left(\frac{1}{3}\left|\boldsymbol{y}_{1}-\boldsymbol{y}_{1}^{\prime}-\frac{1}{2} \boldsymbol{x}\right|^{2}+\frac{1}{4}|\boldsymbol{x}|^{2}\right)} \\
& \times \check{\chi}\left(\omega, \boldsymbol{y}_{1}\right) \bar{\chi}\left(\omega, \boldsymbol{y}_{1}^{\prime}\right) d \boldsymbol{y}_{1} d \boldsymbol{y}_{1}^{\prime} \text {. }
\end{aligned}
$$


Using the first inequality in (8.2) we obtain

$$
\begin{aligned}
\check{\Lambda}_{\mathrm{t}}^{+}\left(\omega, \boldsymbol{x} ; \boldsymbol{x}_{1}, z\right) & \simeq \frac{1}{4} \iint\left(\frac{\omega}{2 \pi z}\right)^{d} e^{i \frac{\omega}{z}\left(\boldsymbol{z}_{1}+\frac{1}{2} \boldsymbol{x}-\boldsymbol{x}_{1}\right) \cdot\left(\boldsymbol{z}_{2}+\boldsymbol{x}\right)} e^{-\frac{\gamma \omega^{2} z}{8}\left(\frac{1}{3}\left|\boldsymbol{z}_{2}-\frac{1}{2} \boldsymbol{x}\right|^{2}+\frac{1}{4}|\boldsymbol{x}|^{2}\right)}\left|\check{\chi}\left(\omega, \boldsymbol{z}_{1}\right)\right|^{2} d \boldsymbol{z}_{1} d \boldsymbol{z}_{2} \\
& \simeq \frac{1}{4} \iint\left(\frac{\omega}{2 \pi z}\right)^{d} e^{i \frac{\omega}{z}\left(\boldsymbol{z}_{1}-\boldsymbol{x}_{1}\right) \cdot\left(\boldsymbol{z}_{2}+\frac{3}{2} \boldsymbol{x}\right)} e^{-\frac{\gamma \omega^{2} z}{8}\left(\frac{1}{3}\left|\boldsymbol{z}_{2}\right|^{2}+\frac{1}{4}|\boldsymbol{x}|^{2}\right)}\left|\check{\chi}\left(\omega, \boldsymbol{z}_{1}\right)\right|^{2} d \boldsymbol{z}_{1} d \boldsymbol{z}_{2} \\
& =\frac{1}{4}\left(\frac{6}{\pi \gamma z^{3}}\right)^{d / 2} \exp \left(-\frac{\gamma \omega^{2} z}{32}|\boldsymbol{x}|^{2}\right) e^{-i \frac{3 \omega}{2 z} \boldsymbol{x}_{1} \cdot \boldsymbol{x}} \int e^{i \frac{3 \omega}{2 z} \boldsymbol{z}_{1} \cdot \boldsymbol{x}} e^{-\frac{6}{\gamma z^{3}}\left|\boldsymbol{z}_{1}-\boldsymbol{x}_{1}\right|^{2}}\left|\check{\chi}\left(\omega, \boldsymbol{z}_{1}\right)\right|^{2} d \boldsymbol{z}_{1} .
\end{aligned}
$$

Then using the second inequality in (8.2) we find

$$
\check{\Lambda}_{\mathrm{t}}^{+}\left(\omega, \boldsymbol{x} ; \boldsymbol{x}_{1}, z\right) \simeq \frac{1}{4}\left(\frac{6}{\pi \gamma z^{3}}\right)^{d / 2} \exp \left(-\frac{\gamma \omega^{2} z}{32}|\boldsymbol{x}|^{2}\right) \exp \left(-\frac{6}{\gamma z^{3}}\left|\boldsymbol{x}_{1}\right|^{2}\right) e^{-i \frac{3 \omega}{2 z} \boldsymbol{x}_{1} \cdot \boldsymbol{x}} \int|\check{\chi}(\omega, \boldsymbol{y})|^{2} d \boldsymbol{y} .
$$

Appendix B. Correlation filter in backscattering case.

We consider the filter $\check{\Lambda}_{\mathrm{t}}^{-}$and derive the representation (10.1). The filter is given by (6.5)

$$
\begin{aligned}
& \check{\Lambda}_{\mathrm{t}}^{-}\left(\omega, \boldsymbol{x} ; \boldsymbol{x}_{1}, z, L\right)=\frac{1}{4} \iint \mathbb{E}\left[\check{\mathcal{T}}\left(\omega,-z, 0, \boldsymbol{y}_{4}, \boldsymbol{y}_{2}\right) \check{\mathcal{T}}\left(\omega,-z, 0, \boldsymbol{y}_{3}, \boldsymbol{y}_{1}\right)\right] \\
& \quad \times \mathbb{E}\left[\check{\mathcal{R}}\left(\omega,-L,-z, \boldsymbol{x}_{1}-\boldsymbol{x}, \boldsymbol{y}_{4}\right) \overline{\mathcal{R}}\left(\omega,-L,-z, \boldsymbol{x}_{1}, \boldsymbol{y}_{3}\right)\right] \check{\chi}\left(\omega, \boldsymbol{y}_{2}\right) \overline{\check{\chi}\left(\omega, \boldsymbol{y}_{1}\right)} d \boldsymbol{y}_{1} d \boldsymbol{y}_{2} d \boldsymbol{y}_{3} d \boldsymbol{y}_{4} \\
& =\iint \mathbb{E}\left[\check{\mathcal{R}}\left(\omega,-L,-z, \boldsymbol{x}_{1}-\boldsymbol{x}, \boldsymbol{y}_{1}\right) \overline{\mathcal{R}}\left(\omega,-L,-z, \boldsymbol{x}_{1}, \boldsymbol{y}_{1}^{\prime}\right)\right] \check{\Lambda}_{\mathrm{t}}^{+}\left(\omega, \boldsymbol{y}_{1}^{\prime}-\boldsymbol{y}_{1} ; \boldsymbol{y}_{1}^{\prime}, z\right) d \boldsymbol{y}_{1} d \boldsymbol{y}_{1}^{\prime} .
\end{aligned}
$$

We write in terms of center and offset coordinates for the reflection operator by

$$
\begin{aligned}
\check{\Lambda}_{\mathrm{t}}^{-}(\omega,-\boldsymbol{y} ; \boldsymbol{x}-\boldsymbol{y} / 2, z, L)= & \iint \mathbb{E}\left[\check{\mathcal{R}}\left(\omega,-L,-z, \boldsymbol{x}+\boldsymbol{y} / 2, \boldsymbol{y}_{1}\right) \overline{\mathcal{R}}\left(\omega,-L,-z, \boldsymbol{x}-\boldsymbol{y} / 2, \boldsymbol{y}_{1}^{\prime}\right)\right] \\
& \times \check{\Lambda}_{\mathrm{t}}^{+}\left(\omega, \boldsymbol{y}_{1}^{\prime}-\boldsymbol{y}_{1} ; \boldsymbol{y}_{1}^{\prime}, z\right) d \boldsymbol{y}_{1} d \boldsymbol{y}_{1}^{\prime} \\
= & (2 \pi)^{-2 d} \iint e^{i\left(\boldsymbol{\kappa} \cdot \boldsymbol{y}+\boldsymbol{\kappa}^{\prime} \cdot\left(\boldsymbol{y}_{1}-\boldsymbol{y}_{1}^{\prime}\right)\right)} W_{\omega}^{\mathrm{R}}\left(L-z, \boldsymbol{x},\left(\boldsymbol{y}_{1}+\boldsymbol{y}_{1}^{\prime}\right) / 2, \boldsymbol{\kappa}, \boldsymbol{\kappa}^{\prime}\right) \\
& \times \check{\Lambda}_{\mathrm{t}}^{+}\left(\omega, \boldsymbol{y}_{1}^{\prime}-\boldsymbol{y}_{1} ; \boldsymbol{y}_{1}^{\prime}, z\right) d \boldsymbol{\kappa} d \boldsymbol{\kappa}^{\prime} d \boldsymbol{y}_{1} d \boldsymbol{y}_{1}^{\prime} .
\end{aligned}
$$

From (C.1) and (C.2) we have

$$
\begin{aligned}
W_{\omega}^{\mathrm{R}}\left(z, \boldsymbol{x}, \boldsymbol{x}^{\prime}, \boldsymbol{\kappa}, \boldsymbol{\kappa}^{\prime}\right)= & (l / 2)^{d} \int e^{i \boldsymbol{\kappa}^{\prime \prime} \cdot\left(\boldsymbol{x}^{\prime}-\boldsymbol{x}\right)} e^{i z\left(\boldsymbol{\kappa}-\boldsymbol{\kappa}^{\prime}\right) \cdot \boldsymbol{\kappa}^{\prime \prime} / \omega} \\
& \times \mathcal{V}^{\mathrm{R}}\left(1, l\left(\boldsymbol{\kappa}+\boldsymbol{\kappa}^{\prime}\right) / 2, l\left(\boldsymbol{\kappa}-\boldsymbol{\kappa}^{\prime}\right), l \boldsymbol{\kappa}^{\prime \prime} ; \alpha(\omega, z), \beta(\omega, z)\right) d \boldsymbol{\kappa}^{\prime \prime},
\end{aligned}
$$

with $\alpha(\omega, z)=z /\left(\omega l^{2}\right)$ characterizing diffractive effects and $\beta(\omega, z)=z \sigma^{2} \omega^{2} l / 4$ characterizing strength of forward scattering. We then get

$$
\begin{aligned}
& \check{\Lambda}_{\mathrm{t}}^{-}(\omega,-\boldsymbol{y} ; \boldsymbol{x}-\boldsymbol{y} / 2, z, L)=(2 \pi)^{-2 d}(l / 2)^{d} \iint e^{i\left(\boldsymbol{\kappa} \cdot \boldsymbol{y}+\boldsymbol{\kappa}^{\prime} \cdot\left(\boldsymbol{y}_{1}-\boldsymbol{y}_{1}^{\prime}\right)\right)} e^{i \boldsymbol{\kappa}^{\prime \prime} \cdot\left(\left(\boldsymbol{y}_{1}+\boldsymbol{y}_{1}^{\prime}\right) / 2-\boldsymbol{x}\right)} e^{i(L-z)\left(\boldsymbol{\kappa}-\boldsymbol{\kappa}^{\prime}\right) \cdot \boldsymbol{\kappa}^{\prime \prime} / \omega} \\
& \quad \times \mathcal{V}^{\mathrm{R}}\left(1, l\left(\boldsymbol{\kappa}+\boldsymbol{\kappa}^{\prime}\right) / 2, l\left(\boldsymbol{\kappa}-\boldsymbol{\kappa}^{\prime}\right), l \boldsymbol{\kappa}^{\prime \prime} ; \alpha(\omega, L-z), \beta(\omega, L-z)\right) \check{\Lambda}_{\mathrm{t}}^{+}\left(\omega, \boldsymbol{y}_{1}^{\prime}-\boldsymbol{y}_{1} ; \boldsymbol{y}_{1}^{\prime}, z\right) d \boldsymbol{\kappa}^{\prime \prime} d \boldsymbol{\kappa} d \boldsymbol{\kappa}^{\prime} d \boldsymbol{y}_{1} d \boldsymbol{y}_{1}^{\prime} \\
& \quad=(2 \pi)^{-2 d}\left(1 /\left(2 l^{2}\right)\right)^{d} \iint e^{i\left((\boldsymbol{q}+\boldsymbol{r} / 2) \cdot \boldsymbol{y} / l+(\boldsymbol{q}-\boldsymbol{r} / 2) \cdot\left(\boldsymbol{y}_{1}-\boldsymbol{y}_{1}^{\prime}\right) / l\right)} e^{i \boldsymbol{s} \cdot\left(\left(\boldsymbol{y}_{1}+\boldsymbol{y}_{1}^{\prime}\right) / 2-\boldsymbol{x}\right) / l} e^{i(L-z) \boldsymbol{r} \cdot \boldsymbol{s} /\left(\omega l^{2}\right)} \\
& \quad \times \mathcal{V}^{\mathrm{R}}(1, \boldsymbol{q}, \boldsymbol{r}, \boldsymbol{s} ; \alpha(\omega, L-z), \beta(\omega, L-z)) \check{\Lambda}_{\mathrm{t}}^{+}\left(\omega, \boldsymbol{y}_{1}^{\prime}-\boldsymbol{y}_{1} ; \boldsymbol{y}_{1}^{\prime}, z\right) d \boldsymbol{q} d \boldsymbol{r} d \boldsymbol{s} d \boldsymbol{y}_{1} d \boldsymbol{y}_{1}^{\prime} .
\end{aligned}
$$

Taking the Fourier transform of the filter in its second and third variables we get

$$
\begin{aligned}
& \check{\Lambda}_{\mathrm{t}}^{-}(\omega,-\boldsymbol{y} ; \boldsymbol{x}-\boldsymbol{y} / 2, z, L)=\left((2 \pi)^{2} 2 l^{2}\right)^{-d} \iint e^{i((\boldsymbol{q}+\boldsymbol{r} / 2) \cdot \boldsymbol{y} / l} e^{-i \boldsymbol{s} \cdot \boldsymbol{x} / l} e^{i \alpha(\omega, L-z) \boldsymbol{r} \cdot \boldsymbol{s}} \\
& \quad \times \mathcal{V}^{\mathrm{R}}(1, \boldsymbol{q}, \boldsymbol{r}, \boldsymbol{s} ; \alpha(\omega, L-z), \beta(\omega, L-z)) \hat{\Lambda}_{\mathrm{t}}^{+}(\omega,(\boldsymbol{r} / 2-\boldsymbol{s} / 2-\boldsymbol{q}) / l ; \boldsymbol{s} / l, z) d \boldsymbol{q} d \boldsymbol{r} d \boldsymbol{s},
\end{aligned}
$$


with

$$
\hat{\Lambda}_{\mathrm{t}}^{+}\left(\omega, \boldsymbol{\kappa}_{1} ; \boldsymbol{\kappa}_{2}, z\right)=\iint \check{\Lambda}_{\mathrm{t}}^{+}\left(\omega, \boldsymbol{y}_{1} ; \boldsymbol{y}_{2}, z\right) e^{i\left(\boldsymbol{\kappa}_{1} \cdot \boldsymbol{y}_{1}+\boldsymbol{\kappa}_{2} \cdot \boldsymbol{y}_{2}\right)} d \boldsymbol{y}_{1} d \boldsymbol{y}_{2}
$$

Using the change of variables $\boldsymbol{s} \mapsto \boldsymbol{s} /(2 \alpha)$ and $\boldsymbol{r} \mapsto \boldsymbol{r}+2 \boldsymbol{q}$ we get

$$
\begin{aligned}
& \check{\Lambda}_{\mathrm{t}}^{-}(\omega,-\boldsymbol{y} ; \boldsymbol{x}-\boldsymbol{y} / 2, z, L)=\left((4 \pi)^{2} \alpha(\omega, L-z) l^{2}\right)^{-d} \iint e^{i((2 \boldsymbol{q}+\boldsymbol{r} / 2) \cdot \boldsymbol{y} / l} e^{-i \boldsymbol{s} \cdot \boldsymbol{x} /(2 l \alpha(\omega, L-z))} \\
& \quad \times e^{i(\boldsymbol{r} / 2+\boldsymbol{q}) \cdot \boldsymbol{s}} \mathcal{V}^{\mathrm{R}}\left(1, \boldsymbol{q}, \boldsymbol{r}+2 \boldsymbol{q}, \frac{\boldsymbol{s}}{2 \alpha(\omega, L-z)} ; \alpha(\omega, L-z), \beta(\omega, L-z)\right) \\
& \quad \times \hat{\Lambda}_{\mathrm{t}}^{+}(\omega,(\boldsymbol{r} / 2-\boldsymbol{s} /(4 \alpha(\omega, L-z))) / l ; \boldsymbol{s} /(2 l \alpha(\omega, L-z)), z) d \boldsymbol{q} d \boldsymbol{r} d \boldsymbol{s} .
\end{aligned}
$$

Next we use Lemma C.1 to get

$$
\begin{aligned}
& \check{\Lambda}_{\mathrm{t}}^{-}(\omega,-\boldsymbol{y} ; \boldsymbol{x}-\boldsymbol{y} / 2, z, L)=\left(4(2 \pi)^{3} \alpha(\omega, L-z) l^{2}\right)^{-d} \iint e^{i((2 \boldsymbol{q}+\boldsymbol{r} / 2) \cdot \boldsymbol{y} / l} e^{-i \boldsymbol{s} \cdot \boldsymbol{x} /(2 l \alpha(\omega, L-z))} \\
& \quad \times e^{i(\boldsymbol{r} / 2+\boldsymbol{q}) \cdot \boldsymbol{s}} e^{-i \boldsymbol{q} \cdot \boldsymbol{u}} e^{\beta(\omega, L-z) \int_{-1}^{1} D_{0}\left(\frac{u}{2}+\frac{s}{2} \zeta\right)-D_{0}(\mathbf{0}) d \zeta} \\
& \quad \times \hat{\Lambda}_{\mathrm{t}}^{+}(\omega,(\boldsymbol{r} / 2-\boldsymbol{s} /(4 \alpha(\omega, L-z))) / l ; \boldsymbol{s} /(2 l \alpha(\omega, L-z)), z) d \boldsymbol{q} d \boldsymbol{r} d \boldsymbol{s} d \boldsymbol{u} .
\end{aligned}
$$

Integrating in $\boldsymbol{q}$ and evaluating the resulting Dirac distribution we get

$$
\begin{aligned}
& \check{\Lambda}_{\mathrm{t}}^{-}(\omega,-\boldsymbol{y} ; \boldsymbol{x}-\boldsymbol{y} / 2, z, L)=\left(4(2 \pi)^{2} \alpha(\omega, L-z) l^{2}\right)^{-d} \iint e^{i \boldsymbol{r} \cdot(\boldsymbol{s}+\boldsymbol{y} / l) / 2} e^{-i \boldsymbol{s} \cdot \boldsymbol{x} /(2 l \alpha(\omega, L-z))} \\
& \quad \times e^{2 \beta \int_{0}^{1} D_{0}(\boldsymbol{y} / l+\boldsymbol{s} \zeta)-D_{0}(\mathbf{0}) d \zeta} \\
& \quad \times \hat{\Lambda}_{\mathrm{t}}^{+}(\omega,(\boldsymbol{r} / 2-\boldsymbol{s} /(4 \alpha(\omega, L-z))) / l ; \boldsymbol{s} /(2 l \alpha(\omega, L-z)), z) d \boldsymbol{r} d \boldsymbol{s} .
\end{aligned}
$$

Finally, we then get

$$
\begin{aligned}
& \check{\Lambda}_{\mathrm{t}}^{-}(\omega,-\boldsymbol{y} ; \boldsymbol{x}-\boldsymbol{y} / 2, z, L)=(2 \pi 2 \alpha(\omega, L-z) l)^{-d} \int e^{\frac{i|s|^{2}}{4 \alpha(\omega, L-z)}} e^{-\frac{i s \cdot(\boldsymbol{x}-\boldsymbol{y} / 2)}{2 l \alpha(\omega, L-z)}} \\
& \quad \times e^{2 \beta \int_{0}^{1} D_{0}\left(\frac{\boldsymbol{y}}{l}+\boldsymbol{s} \zeta\right)-D_{0}(\mathbf{0}) d \zeta} \tilde{\Lambda}_{\mathrm{t}}^{+}\left(\omega,-l \boldsymbol{s}-\boldsymbol{y} ; \frac{\boldsymbol{s}}{2 l \alpha(\omega, L-z)}, z\right) d \boldsymbol{s}
\end{aligned}
$$

for

$$
\tilde{\Lambda}_{\mathrm{t}}^{+}\left(\omega, \boldsymbol{y}_{1} ; \boldsymbol{\kappa}_{2}, z\right)=\int \check{\Lambda}_{\mathrm{t}}^{+}\left(\omega, \boldsymbol{y}_{2} ; \boldsymbol{y}_{2}, z\right) e^{i \boldsymbol{\kappa}_{2} \cdot \boldsymbol{y}_{2}} d \boldsymbol{y}_{2}=\frac{1}{(2 \pi)^{d}} \int \hat{\Lambda}_{\mathrm{t}}^{+}\left(\omega, \boldsymbol{\kappa}_{1} ; \boldsymbol{\kappa}_{2}, z\right) e^{-i \boldsymbol{\kappa}_{1} \cdot \boldsymbol{y}_{2}} d \boldsymbol{\kappa}_{1} \text {. }
$$

We next show that this expression for the filter in fact could have been obtained by using the statistics for the transmission operator rather than those of the reflection operator, however, at a scaled depth. Specifically, the "effective depth" is doubled corresponding to the two way "independent" passages of the bottom part of the slab. Albeit very intuitive, this is a deep result that builds on the Itô-Schrödinger equations and the specific scaling assumptions that we make. It is not valid in general. Important physical phenomena from the point of view of imaging correspond exactly to the situation when this "independence" property fails. The significance of such configurations in our setting will be treated elsewhere. In view of (B.1) we then define

$$
\begin{aligned}
& \check{\mathcal{H}}_{\mathrm{t}}^{-}\left(\omega, \boldsymbol{x} ; \boldsymbol{x}_{1}, z, L\right)=\frac{1}{4} \iint \mathbb{E}\left[\check{\mathcal{T}}\left(\omega,-z, 0, \boldsymbol{y}_{4}, \boldsymbol{y}_{2}\right) \overline{\mathcal{T}}\left(\omega,-z, 0, \boldsymbol{y}_{3}, \boldsymbol{y}_{1}\right)\right] \\
& \quad \times \mathbb{E}\left[\check{\mathcal{T}}\left(\omega,-2 L+z,-z, \boldsymbol{x}_{1}-\boldsymbol{x}, \boldsymbol{y}_{4}\right) \check{\mathcal{T}}\left(\omega,-2 L+z,-z, \boldsymbol{x}_{1}, \boldsymbol{y}_{3}\right)\right] \check{\chi}\left(\omega, \boldsymbol{y}_{2}\right) \overline{\check{\chi}\left(\omega, \boldsymbol{y}_{1}\right)} d \boldsymbol{y}_{1} d \boldsymbol{y}_{2} d \boldsymbol{y}_{3} d \boldsymbol{y}_{4} \\
& =\iint \mathbb{E}\left[\check{\mathcal{T}}\left(\omega,-2 L+z,-z, \boldsymbol{x}_{1}-\boldsymbol{x}, \boldsymbol{y}_{1}\right) \check{\mathcal{T}}\left(\omega,-2 L+z,-z, \boldsymbol{x}_{1}, \boldsymbol{y}_{1}^{\prime}\right)\right] \check{\Lambda}_{\mathrm{t}}^{+}\left(\omega, \boldsymbol{y}_{1}^{\prime}-\boldsymbol{y}_{1} ; \boldsymbol{y}_{1}^{\prime}, z\right) d \boldsymbol{y}_{1} d \boldsymbol{y}_{2} d \boldsymbol{y}_{1} d \boldsymbol{y}_{1}^{\prime}
\end{aligned}
$$


Again we write in terms of center and offset coordinates by

$$
\begin{aligned}
& \check{\mathcal{H}}_{\mathrm{t}}^{-}(\omega,-\boldsymbol{y} ; \boldsymbol{x}-\boldsymbol{y} / 2, z, L)=\iint \mathbb{E}\left[\check{\mathcal{T}}\left(\omega,-2 L+z,-z, \boldsymbol{x}+\boldsymbol{y} / 2, \boldsymbol{y}_{1}\right) \check{\mathcal{T}}\left(\omega,-2 L+z,-z, \boldsymbol{x}-\boldsymbol{y} / 2, \boldsymbol{y}_{1}^{\prime}\right)\right] \\
& \quad \times \check{\Lambda}_{\mathrm{t}}^{+}\left(\omega, \boldsymbol{y}_{1}^{\prime}-\boldsymbol{y}_{1} ; \boldsymbol{y}_{1}^{\prime}, z\right) d \boldsymbol{y}_{1} d \boldsymbol{y}_{1}^{\prime} \\
& =(2 \pi)^{-2 d} \iint e^{i\left(\boldsymbol{\kappa} \cdot \boldsymbol{y}+\boldsymbol{\kappa}^{\prime} \cdot\left(\boldsymbol{y}_{1}-\boldsymbol{y}_{1}^{\prime}\right)\right)} W_{\omega}^{\mathrm{T}}\left(2(L-z), \boldsymbol{x},\left(\boldsymbol{y}_{1}+\boldsymbol{y}_{1}^{\prime}\right) / 2, \boldsymbol{\kappa}, \boldsymbol{\kappa}^{\prime}\right) \\
& \quad \times \check{\Lambda}_{\mathrm{t}}^{+}\left(\omega, \boldsymbol{y}_{1}^{\prime}-\boldsymbol{y}_{1} ; \boldsymbol{y}_{1}^{\prime}, z\right) d \boldsymbol{\kappa} d \boldsymbol{\kappa}^{\prime} d \boldsymbol{y}_{1} d \boldsymbol{y}_{1}^{\prime} .
\end{aligned}
$$

Using (7.1) we then get

$$
\begin{aligned}
\check{\mathcal{H}}_{\mathrm{t}}^{-}(\omega, & -\boldsymbol{y} ; \boldsymbol{x}-\boldsymbol{y} / 2, z, L)=(2 \pi)^{-3 d} \iint e^{i\left(\boldsymbol{\kappa} \cdot \boldsymbol{y}+\boldsymbol{\kappa}^{\prime} \cdot\left(\boldsymbol{y}_{1}-\boldsymbol{y}_{1}^{\prime}\right)\right)} e^{-i\left(\boldsymbol{\kappa}^{\prime}+\boldsymbol{\kappa}\right) \cdot \boldsymbol{a}-i\left(\left(\boldsymbol{y}_{1}+\boldsymbol{y}_{1}^{\prime}\right) / 2-\boldsymbol{x}+\frac{\boldsymbol{\kappa}}{\omega} z^{*}\right) \cdot \boldsymbol{b}} \\
& \times e^{\frac{\omega^{2}}{4} \int_{0}^{z^{*}} D\left(\boldsymbol{a}+\frac{\boldsymbol{b}}{\omega} z^{\prime}\right)-D(\mathbf{0}) d z^{\prime}} d \boldsymbol{a} d \boldsymbol{b} \check{\Lambda}_{\mathrm{t}}^{+}\left(\omega, \boldsymbol{y}_{1}^{\prime}-\boldsymbol{y}_{1} ; \boldsymbol{y}_{1}^{\prime}, z\right) d \boldsymbol{\kappa} d \boldsymbol{\kappa}^{\prime} d \boldsymbol{y}_{1} d \boldsymbol{y}_{1}^{\prime} \\
= & (2 \pi)^{-3 d} \iint e^{i\left(\boldsymbol{\kappa} \cdot\left(\boldsymbol{y}-\boldsymbol{a}-\frac{z^{*} \boldsymbol{b}}{\omega}\right)\right.} e^{i\left(\boldsymbol{\kappa}^{\prime} \cdot\left(\boldsymbol{y}_{1}-\boldsymbol{y}_{1}^{\prime}-\boldsymbol{a}\right)\right.} e^{-i\left(\frac{\boldsymbol{y}_{1}+\boldsymbol{y}_{1}^{\prime}}{2}-\boldsymbol{x}\right) \cdot \boldsymbol{b}} \\
& \times e^{\beta\left(\omega, z^{*}\right) \int_{0}^{1} D_{0}\left(\frac{\boldsymbol{a}}{l}+\frac{\boldsymbol{b} z^{*}}{\omega l} \zeta\right)-D_{0}(\mathbf{0}) d \zeta} d \boldsymbol{a} d \boldsymbol{b} \check{\Lambda}_{\mathrm{t}}^{+}\left(\omega, \boldsymbol{y}_{1}^{\prime}-\boldsymbol{y}_{1} ; \boldsymbol{y}_{1}^{\prime}, z\right) d \boldsymbol{\kappa} d \boldsymbol{\kappa}^{\prime} d \boldsymbol{y}_{1} d \boldsymbol{y}_{1}^{\prime},
\end{aligned}
$$

for $z^{*}=2(L-z)$. Integrating in $\boldsymbol{\kappa}$ and $\boldsymbol{\kappa}^{\prime}$ and evaluating the resulting two Dirac distributions we get

$$
\begin{aligned}
& \check{\mathcal{H}}_{\mathrm{t}}^{-}(\omega,-\boldsymbol{y} ; \boldsymbol{x}-\boldsymbol{y} / 2, z, L)=(2 \pi)^{-d} \iint e^{-i \boldsymbol{b} \cdot\left(\boldsymbol{y}_{1}^{\prime}+\boldsymbol{y} / 2-\frac{z^{*} \boldsymbol{b}}{2 \omega}\right)} e^{i \boldsymbol{x} \cdot \boldsymbol{b}} \\
& \quad \times e^{\beta\left(\omega, z^{*}\right) \int_{0}^{1} D_{0}\left(\frac{y}{l}-\frac{\boldsymbol{b} z^{*}}{\omega l} \zeta\right)-D_{0}(\mathbf{0}) d \zeta} d \boldsymbol{b} \check{\Lambda}_{\mathrm{t}}^{+}\left(\omega,-\boldsymbol{y}-z^{*} \boldsymbol{b} / \omega ; \boldsymbol{y}_{1}^{\prime}, z\right) d \boldsymbol{y}_{1} d \boldsymbol{y}_{1}^{\prime} .
\end{aligned}
$$

Upon the change of variables $\boldsymbol{b} \mapsto-s \omega l / z^{*}$ we get:

$$
\begin{aligned}
\check{\mathcal{H}}_{\mathrm{t}}^{-}(\omega, & -\boldsymbol{y} ; \boldsymbol{x}-\boldsymbol{y} / 2, z, L)=\frac{1}{\left(2 \pi \alpha\left(\omega, z^{*}\right) l\right)^{d}} \iint e^{i \frac{\boldsymbol{s} \cdot\left(\boldsymbol{y}_{1}^{\prime}+\boldsymbol{y} / 2\right)}{l \alpha\left(\omega, z^{*}\right)}} e^{\frac{i|\boldsymbol{s}|^{2}}{2 \alpha\left(\omega, z^{*}\right)}} e^{-\frac{i \boldsymbol{x} \cdot \mathbf{s}}{l \alpha\left(\omega, z^{*}\right)}} \\
& \times e^{\beta\left(\omega, z^{*}\right) \int_{0}^{1} D_{0}\left(\frac{y}{l}+\boldsymbol{s} \zeta\right)-D_{0}(\mathbf{0}) d \zeta} \check{\Lambda}_{\mathrm{t}}^{+}\left(\omega,-\boldsymbol{y}-l \boldsymbol{s} ; \boldsymbol{y}_{1}^{\prime}, z\right) d \boldsymbol{s} d \boldsymbol{y}_{1}^{\prime} \\
= & \frac{1}{\left(2 \pi l \alpha\left(\omega, z^{*}\right)\right)^{d}} \iint e^{\frac{i|\boldsymbol{s}|^{2}}{2 \alpha\left(\omega, z^{*}\right)}} e^{-\frac{i(\boldsymbol{x}-\boldsymbol{y} / 2) \cdot \mathbf{s}}{l \alpha\left(\omega, z^{*}\right)}} \\
& \times e^{\beta\left(\omega, z^{*}\right) \int_{0}^{1} D_{0}\left(\frac{y}{l}+\boldsymbol{s} \zeta\right)-D_{0}(\mathbf{0}) d \zeta} \tilde{\Lambda}_{\mathrm{t}}^{+}\left(\omega,-\boldsymbol{y}-l \boldsymbol{s} ; \frac{\boldsymbol{s}}{l \alpha\left(\omega, z^{*}\right)}, z\right) d \boldsymbol{s} .
\end{aligned}
$$

Then, by comparing with (B.2) and observing that $\alpha\left(\omega, z^{*}\right)=2 \alpha(\omega, L-z), \beta\left(\omega, z^{*}\right)=2 \beta(\omega, L-z)$ we conclude $\check{\mathcal{H}}_{\mathrm{t}}^{-}(\omega,-\boldsymbol{y} ; \boldsymbol{x}-\boldsymbol{y} / 2, z, L)=\check{\Lambda}_{\mathrm{t}}^{-}(\omega,-\boldsymbol{y} ; \boldsymbol{x}-\boldsymbol{y} / 2, z, L)$. We therefore have

$$
\begin{aligned}
\check{\Lambda}_{\mathrm{t}}^{-} & \left(\omega, \boldsymbol{x} ; \boldsymbol{x}_{1}, z, L\right)=\frac{1}{4} \iint \mathbb{E}\left[\check{\mathcal{T}}\left(\omega,-z, 0, \boldsymbol{y}_{4}, \boldsymbol{y}_{2}\right) \overline{\mathcal{T}}\left(\omega,-z, 0, \boldsymbol{y}_{3}, \boldsymbol{y}_{1}\right)\right] \\
& \times \mathbb{E}\left[\check{\mathcal{R}}\left(\omega,-L,-z, \boldsymbol{x}_{1}-\boldsymbol{x}, \boldsymbol{y}_{4}\right) \overline{\mathcal{\mathcal { R }}\left(\omega,-L,-z, \boldsymbol{x}_{1}, \boldsymbol{y}_{3}\right)}\right] \check{\chi}\left(\omega, \boldsymbol{y}_{2}\right) \overline{\check{\chi}\left(\omega, \boldsymbol{y}_{1}\right)} d \boldsymbol{y}_{1} d \boldsymbol{y}_{2} d \boldsymbol{y}_{3} d \boldsymbol{y}_{4} \\
= & \frac{1}{4} \iint \mathbb{E}\left[\check{\mathcal{T}}\left(\omega,-z, 0, \boldsymbol{y}_{4}, \boldsymbol{y}_{2}\right) \overline{\mathcal{T}}\left(\omega,-z, 0, \boldsymbol{y}_{3}, \boldsymbol{y}_{1}\right)\right] \\
& \times \mathbb{E}\left[\check{\mathcal{T}}\left(\omega,-2 L+z,-z, \boldsymbol{x}_{1}-\boldsymbol{x}, \boldsymbol{y}_{4}\right) \overline{\mathcal{T}}\left(\omega,-2 L+z,-z, \boldsymbol{x}_{1}, \boldsymbol{y}_{3}\right)\right] \check{\chi}\left(\omega, \boldsymbol{y}_{2}\right) \overline{\check{\chi}\left(\omega, \boldsymbol{y}_{1}\right)} d \boldsymbol{y}_{1} d \boldsymbol{y}_{2} d \boldsymbol{y}_{3} d \boldsymbol{y}_{4} \\
= & \frac{1}{4} \iint \mathbb{E}\left[\check{\mathcal{T}}\left(\omega,-2 L+z, 0, \boldsymbol{x}_{1}-\boldsymbol{x}, \boldsymbol{y}_{2}\right) \overline{\mathcal{T}}\left(\omega,-2 L+z, 0, \boldsymbol{x}_{1}, \boldsymbol{y}_{1}\right)\right] \check{\chi}\left(\omega, \boldsymbol{y}_{2}\right) \bar{\chi}\left(\omega, \boldsymbol{y}_{1}\right) d \boldsymbol{y}_{1} d \boldsymbol{y}_{2} \\
= & \check{\Lambda}_{\mathrm{t}}^{+}\left(\omega, \boldsymbol{x} ; \boldsymbol{x}_{1}, 2 L-z\right) .
\end{aligned}
$$

\section{Appendix C. Wigner asymtotics.}

We cast the Wigner distribution in a suitable dimensionless form and present an asymptotic approximation valid in regime given by the scaling relation in Assumption 3. In this case we have 
$l \ll r_{0}$, and relatively rapid medium fluctuations. Moreover, we have $\omega l r_{0}=\mathcal{O}(z)$ which means that diffractive effects are of order one at depth $z$. This scaling assumption is equivalent to

$$
\alpha_{0}=\frac{z}{\omega r_{0}^{2}} \ll \alpha_{e}=\frac{z}{\omega l r_{0}}=\mathcal{O}(1) \ll \alpha=\alpha(\omega, z)=\frac{z}{\omega l^{2}} .
$$

The parameters $\alpha_{0}, \alpha_{e}, \alpha$ are inverse Fresnel numbers with the aperture corresponding respectively to the source aperture $r_{0}$, effective aperture $\sqrt{l r_{0}}$ and the medium correlation length $l$. They describe strength of diffractive effects for respectively homogeneous medium with source aperture $r_{0}$, the random medium with again source aperture $r_{0}$ and the homogeneous medium with source aperture $l$.

We consider the following Fourier transform $V^{\mathrm{R}}$ of the Wigner distribution $W_{\omega}^{\mathrm{R}}$ :

$$
W_{\omega}^{\mathrm{R}}\left(z, \boldsymbol{x}, \boldsymbol{x}^{\prime}, \boldsymbol{\kappa}, \boldsymbol{\kappa}^{\prime}\right)=\frac{1}{(2 \pi)^{d}} \int V_{\omega}^{\mathrm{R}}\left(z, \frac{\boldsymbol{\kappa}+\boldsymbol{\kappa}^{\prime}}{2}, \boldsymbol{\kappa}-\boldsymbol{\kappa}^{\prime}, \boldsymbol{\kappa}^{\prime \prime}\right) e^{i \boldsymbol{\kappa}^{\prime \prime} \cdot\left(\boldsymbol{x}^{\prime}-\boldsymbol{x}\right)} d \boldsymbol{\kappa}^{\prime \prime},
$$

which we introduce because the stationary maps that we will identify in Lemma C.1, in the asymptotic regime $\alpha \rightarrow \infty$, have simple representations in this new frame. Note also that this ansatz incorporates the fact that $W_{\omega}^{\mathrm{R}}$ does not depend on $\boldsymbol{x}+\boldsymbol{x}^{\prime}$, only on $\boldsymbol{x}-\boldsymbol{x}^{\prime}, \boldsymbol{\kappa}$, and $\boldsymbol{\kappa}^{\prime}$, which follows from the stationarity of the random medium. The Fourier-transformed operator $V_{\omega}^{\mathrm{R}}\left(z, \boldsymbol{\kappa}, \boldsymbol{\kappa}^{\prime}, \boldsymbol{\kappa}^{\prime \prime}\right)$ has the form

$$
V_{\omega}^{\mathrm{R}}\left(z, \boldsymbol{\kappa}, \boldsymbol{\kappa}^{\prime}, \boldsymbol{\kappa}^{\prime \prime}\right)=(\pi l)^{d} e^{\frac{i z}{\omega} \boldsymbol{\kappa}^{\prime} \cdot \boldsymbol{\kappa}^{\prime \prime}} \mathcal{V}^{\mathrm{R}}\left(1, \boldsymbol{\kappa} l, \boldsymbol{\kappa}^{\prime} l, \boldsymbol{\kappa}^{\prime \prime} l ; \alpha(\omega, z), \beta(\omega, z)\right),
$$

where $\left(\mathcal{V}^{\mathrm{R}}(\zeta, \boldsymbol{q}, \boldsymbol{r}, \boldsymbol{s} ; \alpha, \beta)\right)_{\zeta \in[0,1]}$ is the solution of the dimensionless system

$$
\begin{aligned}
\frac{\partial \mathcal{V}^{\mathrm{R}}}{\partial \zeta}= & \frac{\beta}{(2 \pi)^{d}} \int \hat{D}_{0}(\boldsymbol{u})\left[\mathcal{V}^{\mathrm{R}}\left(\zeta, \boldsymbol{q}-\frac{1}{2} \boldsymbol{u}, \boldsymbol{r}-\boldsymbol{u}, \boldsymbol{s}\right) e^{-i \alpha \boldsymbol{s} \cdot \boldsymbol{u} \zeta}\right. \\
& +\mathcal{V}^{\mathrm{R}}\left(\zeta, \boldsymbol{q}-\frac{1}{2} \boldsymbol{u}, \boldsymbol{r}+\boldsymbol{u}, \boldsymbol{s}\right) e^{i \alpha \boldsymbol{s} \cdot \boldsymbol{u} \zeta}+\mathcal{V}^{\mathrm{R}}\left(\zeta, \boldsymbol{q}-\frac{1}{2} \boldsymbol{u}, \boldsymbol{r}, \boldsymbol{s}-\boldsymbol{u}\right) e^{-i \alpha \boldsymbol{r} \cdot \boldsymbol{u} \zeta} \\
& +\mathcal{V}^{\mathrm{R}}\left(\zeta, \boldsymbol{q}-\frac{1}{2} \boldsymbol{u}, \boldsymbol{r}, \boldsymbol{s}+\boldsymbol{u}\right) e^{i \alpha \boldsymbol{r} \cdot \boldsymbol{u} \zeta}-2 \mathcal{V}^{\mathrm{R}}(\zeta, \boldsymbol{\kappa}, \boldsymbol{r}, \boldsymbol{s}) \\
& -\mathcal{V}^{\mathrm{R}}\left(\zeta, \boldsymbol{q}-\frac{1}{2} \boldsymbol{u}, \boldsymbol{r}-\boldsymbol{u}, \boldsymbol{s}+\boldsymbol{u}\right) e^{i \alpha\left[(\boldsymbol{r}-\boldsymbol{s}) \cdot \boldsymbol{u}-|\boldsymbol{u}|^{2}\right] \zeta} \\
& \left.-\mathcal{V}^{\mathrm{R}}\left(\zeta, \boldsymbol{q}-\frac{1}{2} \boldsymbol{u}, \boldsymbol{r}-\boldsymbol{u}, \boldsymbol{s}-\boldsymbol{u}\right) e^{-i \alpha\left[(\boldsymbol{r}+\boldsymbol{s}) \cdot \boldsymbol{u}+|\boldsymbol{u}|^{2}\right] \zeta}\right] d \boldsymbol{u},
\end{aligned}
$$

starting from $\mathcal{V}^{\mathrm{R}}(\zeta=0, \boldsymbol{q}, \boldsymbol{r}, \boldsymbol{s} ; \alpha, \beta)=\delta(\boldsymbol{q})$. Recall that $\alpha(\omega, z)=z /\left(\omega l^{2}\right)$ and $\beta(\omega, z)=\sigma^{2} \omega^{2} l z / 4$.

The rapid transverse variations regime is particularly interesting to study because $W_{\omega}^{\mathrm{R}}$ has a multi-scale behavior. In (C.3) this regime gives rise to rapid phases and this allows us to identify a simplified description and the multiscale behavior strongly influences the correlations. The following lemma describes the asymptotic behavior of $\mathcal{V}^{\mathrm{R}}$ as $\alpha \rightarrow \infty$. The presence of singular layers at $\boldsymbol{r}=\mathbf{0}$ and at $\boldsymbol{s}=\mathbf{0}$ requires particular attention and is responsible for instance for the enhanced backscattering phenomenon [6], (corresponding to part (3) in Lemma C.1). In general (part (1) in Lemma C.1) the intensity of the reflection operator decays exponentially according to the parameter $\beta D_{0}(\mathbf{0})$ corresponding to the total scattering cross section. This decay follows from a partial loss of coherence by random forward scattering. However, as articulated in parts (2) and (3) of the lemma below the coupling of wave modes depends on the full medium autocorrelation function if we look at nearby specular reflection and or small spatial offset frequencies. This coupling will be important in the analysis of the correlations in Section 10. We have [6]

Lemma C.1.

(1) For any $\boldsymbol{r} \neq \mathbf{0}, \boldsymbol{s} \neq \mathbf{0}$ :

$$
\mathcal{V}^{\mathrm{R}}(\zeta, \boldsymbol{q}, \boldsymbol{r}, \boldsymbol{s} ; \alpha, \beta) \stackrel{\alpha \rightarrow \infty}{\longrightarrow} \delta(\boldsymbol{q}) e^{-2 \beta D_{0}(\mathbf{0}) \zeta} .
$$


(2) For any $\boldsymbol{s} \neq \mathbf{0}$ we have $\mathcal{V}^{\mathrm{R}}\left(\zeta, \boldsymbol{q}, \frac{\boldsymbol{r}}{\alpha}, \boldsymbol{s} ; \alpha, \beta\right) \stackrel{\alpha \rightarrow \infty}{\longrightarrow} \mathcal{V}_{\boldsymbol{r}}^{\mathrm{R}}(\zeta, \boldsymbol{q} ; \beta)$ where $\mathcal{V}_{\boldsymbol{r}}^{\mathrm{R}}(\zeta, \boldsymbol{q} ; \beta)$ is solution of

$$
\frac{\partial \mathcal{V}_{\boldsymbol{r}}^{\mathrm{R}}}{\partial \zeta}=\frac{2 \beta}{(2 \pi)^{d}} \int \hat{D}_{0}(\boldsymbol{u})\left[\mathcal{V}_{\boldsymbol{r}}^{\mathrm{R}}\left(\zeta, \boldsymbol{q}-\frac{1}{2} \boldsymbol{u}\right) \cos (\boldsymbol{r} \cdot \boldsymbol{u} \zeta)-\mathcal{V}_{\boldsymbol{r}}^{\mathrm{R}}(\zeta, \boldsymbol{q})\right] d \boldsymbol{u},
$$

and is given explicitly by

$$
\mathcal{V}_{\boldsymbol{r}}^{\mathrm{R}}(\zeta, \boldsymbol{q} ; \beta)=\frac{1}{(2 \pi)^{d}} \int e^{-i \boldsymbol{q} \cdot \boldsymbol{u}} e^{\beta \int_{0}^{\zeta} D_{0}\left(\frac{\boldsymbol{u}}{2}+\boldsymbol{r} \zeta^{\prime}\right)+D_{0}\left(\frac{\boldsymbol{u}}{2}-\boldsymbol{r} \zeta^{\prime}\right)-2 D_{0}(\mathbf{0}) d \zeta^{\prime}} d \boldsymbol{u} .
$$

Similarly, for any $\boldsymbol{r} \neq \mathbf{0}$ we have $\mathcal{V}^{\mathrm{R}}\left(\zeta, \boldsymbol{q}, \boldsymbol{r}, \frac{\boldsymbol{s}}{\alpha} ; \alpha, \beta\right) \stackrel{\alpha \rightarrow \infty}{\longrightarrow} \mathcal{V}_{\boldsymbol{s}}^{\mathrm{R}}(\zeta, \boldsymbol{q} ; \beta)$.

(3) For any $\boldsymbol{r}$ and $\boldsymbol{s}$ we have

$$
\mathcal{V}^{\mathrm{R}}\left(\zeta, \boldsymbol{q}, \frac{\boldsymbol{r}}{\alpha}, \frac{\boldsymbol{s}}{\alpha} ; \alpha, \beta\right) \stackrel{\alpha \rightarrow \infty}{\longrightarrow} \mathcal{V}_{\boldsymbol{r}}^{\mathrm{R}}(\zeta, \boldsymbol{q} ; \beta)+\mathcal{V}_{\boldsymbol{s}}^{\mathrm{R}}(\zeta, \boldsymbol{q} ; \beta)-\delta(\boldsymbol{q}) e^{-2 \beta D_{0}(\mathbf{0}) \zeta}
$$

\section{REFERENCES}

[1] C. Bardos, J. Garnier, and G. Papanicolaou, Identification of Green's function singularities by cross correlation of noisy signals, Inv. Problems, 24, 015011 (2008).

[2] M. Campillo and A. Paul, Long-range correlations in the diffuse seismic coda, Science, 299, 547-549 (2003).

[3] A. Derode, E. Larose, M. Tanter, J. de Rosny, A. Tourin, M. Campillo, and M. Fink, Recovering the Green's function from field-field correlations in an open scattering medium, J. Acoust. Soc. Am., 113, 2973-2976 (2003)

[4] D. Draganov, K. Wapenaar, W. Mulder, J. Singer, and A. Verdel, Retrieval of reflections from seismic background-noise measurements, Geophys. Res. Lett., 34, L04305 (2007).

[5] J. Garnier and G. Papanicolaou, Passive sensor imaging using cross correlations of noisy signals in a scattering medium, SIAM J. Imaging Sciences, 2, 396-437 (2009).

[6] J. Garnier and K. Sølna, Coupled paraxial wave equations in random media in the white-noise regime, Ann. Appl. Probab. 19, 318-346 (2009).

[7] J. Garnier and K. Sølna, Scaling limits for wave pulse transmission and reflection operators, Wave Motion, 2, 122-143 (2009).

[8] M.V. de Hoop and K. Sølna, Estimating a Green's function from "field-field" correlations in a random medium, SIAM J. Appl. Math., 69, 909-932 (2009).

[9] P. Roux, K. G. Sabra, P. Gerstoft, W. A. Kuperman, and M. Fehler, P-waves from cross-correlation of seismic noise, Geophys. Res. Lett., 32, L19303 (2005).

[10] N. M. Shapiro, M. Campillo, L. Stehly, and M. Ritzwoller, High-resolution surface-wave tomography from ambient seismic noise, Science, 307, 11, 2005.

[11] B. A. van Tiggelen, Green function retrieval and time reversal in a disordered world, Phys. Rev. Lett., 91, 243904 (2003).

[12] T. Tonegawa, K. Nishida, T. Watanabe, and K. Shiomi, Seismic interferometry of teleseismic S-wave coda for retrieval of body waves: an application to the Philippine Sea slab underneath the Japanese Islands, Geophys. J. Int., 178, 1574-1586 (2009).

[13] K. Wapenaar, Seismic interferometry, the optical theorem and a non-linear diffractor, 79th annual SEG meeting, Houston, 3595-3600 (2009).

[14] R. L. Weaver and O. I. Lobkis, Ultrasonics without a source: Thermal fluctuation correlations at Mhz frequencies, Phys. Rev. Lett., 93, 254301 (2001).

[15] H. Yao, R. D. van der Hilst, and M. V. de Hoop, Surface-wave array tomography in SE Tibet from ambient seismic noise and two-station analysis - I. Phase velocity maps, Geophys. J. Int., 166, 732-744 (2006). 